\title{
Theoretical study of elastic electron scattering off stable and exotic nuclei
}

\author{
X. Roca-Maza, ${ }^{*}$ M. Centelles, F. Salvat, and X. Viñas \\ Departament d'Estructura i Constituents de la Matèria and Institut de Ciències del Cosmos, Facultat de Física, \\ Universitat de Barcelona, Diagonal 647, 08028 Barcelona, Spain
}

(Received 8 August 2008; published 31 October 2008)

\begin{abstract}
Results for elastic electron scattering by nuclei, calculated with charge densities of Skyrme forces and covariant effective Lagrangians that accurately describe nuclear ground states, are compared against experiment in stable isotopes. Dirac partial-wave calculations are performed with an adapted version of the ELSEPA package. Motivated by the fact that studies of electron scattering off exotic nuclei are intended in future facilities in the commissioned GSI and RIKEN upgrades, we survey the theoretical predictions from neutron-deficient to neutron-rich isotopes in the tin and calcium isotopic chains. The charge densities of a covariant interaction that describes the low-energy electromagnetic structure of the nucleon within the Lagrangian of the theory are used to this end. The study is restricted to medium- and heavy-mass nuclei because the charge densities are computed in mean-field approach. Because the experimental analysis of scattering data commonly involves parameterized charge densities, as a surrogate exercise for the yet unexplored exotic nuclei, we fit our calculated mean-field densities with Helm model distributions. This procedure turns out to be helpful to study the neutron-number variation of the scattering observables and allows us to identify correlations of potential interest among some of these observables within the isotopic chains.
\end{abstract}

DOI: 10.1103/PhysRevC.78.044332

PACS number(s): 21.10.Ft, 25.30.Bf, 13.40.Gp, 21.60.-n

\section{INTRODUCTION}

Elastic electron-nucleus scattering has been for many years a very useful tool to investigate the size and shape of stable nuclei [1-5]. Electrons interact with nuclei basically through the electromagnetic force. If the energy of the electrons is high enough, they become a relatively clean probe to explore precisely the internal structure of nuclei, insensitive to strong interaction effects. In particular, the analysis of electron-scattering data provides most valuable information about the charge distribution in atomic nuclei [6-8].

Developments in accelerator technology and detection techniques nowadays allow experimentation with nuclei beyond the limits of $\beta$ stability. The number of nuclei whose masses have been measured keeps growing [9] and this tendency is expected to continue with the use of radioactive isotope beams (RIB) [10-12]. A new generation of electron-RIB colliders using storage rings is now under construction in RIKEN (Japan) $[13,14]$ and GSI (Germany) $[15,16]$. These facilities will offer unprecedented opportunities to study the structure of exotic unstable nuclei through electron scattering in the ELISe experiment at the Facility for Antiproton and Ion Research in Germany [17] and the SCRIT project in Japan $[18,19]$. Therefore, the theoretical investigation of exotic nuclei with models of purported reliability in stable isotopes is a timely and challenging problem. Such effort will test the ability of the established nuclear theory in the domain of exotic nuclei and may as well provide valuable references for future experiments.

In recent literature, several theoretical studies of elastic electron-nucleus scattering in exotic nuclei have been reported [20-26]. Some of these works are concerned with analyzing

\footnotetext{
*roca@ecm.ub.es
}

electron scattering in light nuclei, where exciting exotic phenomena such as the appearence of halos may take place. That is the case of, e.g., ${ }^{6,8} \mathrm{He}[20,22],{ }^{11} \mathrm{Li}[20,23],{ }^{8} \mathrm{~B}[22,23],{ }^{12} \mathrm{O}$, and ${ }^{28} \mathrm{~S}$ [24] nuclei, where possible effects on scattering from the occurrence of halos have been investigated. Light nuclei require a microscopic treatment of the scattering interaction to properly deal with the underlying shell-model structure [22]. Other works study the variation of the charge form factors along isotopic [20,21,25] and isotonic [26] chains of medium and heavy mass nuclei. It has been found that when the number of neutrons (protons) in these isotopic (isotonic) chains increases, the squared modulus of the charge form factor and the position of its minima show, respectively, an upward trend and a significant inward shifting in the momentum transfer. In addition to electron scattering, it is worth mentioning that proton scattering may be another valuable tool to investigate the changes in the charge density of the nucleus, especially at its interior, as one proceeds to the drip lines along isotopic chains [27].

To investigate the internal structure of nuclear charge densities, the de Broglie wavelength of the probe has to be of the order of $1 \mathrm{fm}$. This means that the energy of the electron beam has to be of the order of several hundred MeV. Therefore, for accurate theoretical calculations of differential cross sections (DCS) and electric charge form factors, one needs to solve the elastic scattering of Dirac particles in the scalar potential pertaining to the nuclear charge distribution. The simplest approach is the plane-wave Born approximation (PWBA), where one assumes that the initial and final states of the electron can be described by plane waves. Although the PWBA is able to account for important features of scattering, it is not enough accurate for quantitative calculations of the electric charge form factor. A more elaborate method is supplied by the Glauber theory using a relativistic eikonal approximation of the Dirac equation [28]. It has been successfully applied 
to a systematic study in elastic electron-nucleus scattering [24-26]. The most sophisticated calculations of electronnucleus scattering employ the exact phase-shift analysis of the Dirac equation. This method corresponds to the so-called distorted wave Born approximation (DWBA) [29] and was employed, e.g., in Refs. [20,21]. In the present work we apply a modified version of the recently published code ELSEPA [30] to the elastic electron-nucleus scattering problem. This code was originally devised to perform accurate Dirac partial-wave calculations of elastic scattering of electrons and positrons by atoms, positive ions, and molecules in the low-energy domain.

The main input needed for solving the elastic electronnucleus scattering problem is the charge density of the target nucleus. In the present article we use charge densities obtained with mean-field models for calculating electron scattering off medium- and heavy-mass nuclei. For this purpose, we employ effective nuclear interactions of current use in nuclear structure physics. It is known that the overall trends of the elastic electron-nucleus scattering in medium and heavy nuclei are, in general, reasonably reproduced by the theoretical charge densities obtained in the mean-field approximation. However, different effective interactions predict electric charge form factors and DCS that differ in fine details and describe with different quality the experimental data. In our study we consider the nonrelativistic Skyrme forces SkM* [31] and SLy4 [32] and the covariant models NL3 [33], FSUGold [34], G2 [35], and DD-ME2 [36]. They are representative examples of effective interactions that accurately describe the ground-state properties of finite nuclei and some of their collective excitations.

The parameters of the alluded microscopic interactions have been determined from careful calibration to observables such as binding energies, single-particle levels, and charge and diffraction radii of a variety of selected nuclei. The nucleon density distributions of the Skyrme Hartree-Fock and relativistic mean-field theories are obtained by numerical solution of the quantal mean-field variational equations. The effects of the neutrons on the proton density are taken into account, in a self-consistent manner, through the interaction terms of the effective force or Lagrangian. Therefore, no parametrized shapes of the density profiles are implemented. Nevertheless, most of the mean-field calculations of finite nuclei assume point-nucleon densities. The charge density is obtained from the proton pointlike distribution folded with the proton charge form factor [37]

$$
\rho_{p}(r)=\frac{\alpha^{3}}{8 \pi} e^{-\alpha r} .
$$

A value $\alpha^{2}=18.29 \mathrm{fm}^{-2}$ corresponds to a proton rootmean-square (rms) radius of $0.81 \mathrm{fm}$. This is the standard prescription in the fitting procedure of the parameters of most Skyrme forces and relativistic mean-field interactions to the experimental data of finite nuclei, including charge radii. The covariant models of Ref. [35] are an exception to this fact and, in particular, the interaction $G 2[35,38]$ that we will employ in several of our calculations. The effective Lagrangian of $G 2$ incorporates the low-energy electromagnetic structure of the nucleon within the theory $[35,38]$. It is to be emphasized that in $G 2$ the charge density is obtained directly from the solution of the mean-field equations and that there is no folding to be performed with external single-nucleon form factors, hereby maximizing the predictive power. In the other mean-field forces considered in our work we will neglect the contribution of the neutron charge form factor [39] to the charge density. This is known to be a reasonable approximation up to moderate-momentum transfers [40], which is the region analyzed in the present study of scattering. Moreover, it ensures consistency with the method applied originally to fit the parameters of these interactions; additional modifications into the charge densities could spoil, e.g., their accurate predictions for charge radii.

It is to be mentioned that the mean-field treatment would reach its limits in the study of exotic light nuclei, where the shell-model structure and halos can become prevailing features [22]. Therefore we do not attempt to treat these light systems in the present work. The development of suitable tools and a unified framework to deal with charge densities and electron scattering in nuclei across the mass table remains an outstanding problem in the field, maybe appropriate for new initiatives like the UNEDF collaboration to build a universal nuclear energy density functional [41].

The present article is organized as follows. Section II is devoted to the theoretical formalism. The mean-field description of finite nuclei in the nonrelativistic and relativistic frames is briefly discussed. The Dirac partial-wave calculation of elastic scattering of electrons by nuclei implemented in the code ELSEPA is summarized. In Sec. III, the elastic electron scattering results obtained from the charge densities of the above-mentioned effective interactions are studied by comparing with available experimental data for several stable nuclei. In Sec. IV we investigate the theoretical predictions for elastic electron-nucleus scattering in the tin and calcium isotopic chains from the proton drip line to the neutron drip line. These calculations are performed with the relativistic mean-field interaction $G 2$ [35]. Our conclusions are laid in the final section.

\section{THEORY}

In the current section we review the basic features of the Skyrme and relativistic mean-field models that we will employ to compute the theoretical nuclear charge densities. We also summarize the calculation of Dirac distorted waves for elastic electron-nucleus scattering in the code ELSEPA. The reader conversant with effective nuclear mean-field models and with knowledge of the basics of Dirac partial-wave calculations may prefer to proceed directly to the discussion of results that starts in Sec. III.

\section{A. Mean-field description of nuclei}

The mean-field approach assumes that nucleons move independently in a mean-field generated by the other nucleons of the atomic nucleus. Useful tools for mean-field calculations of nuclei are the nonrelativistic Hartree-Fock method with phenomenological interactions and the relativistic mean-field (RMF) Hartree model with effective Lagrangians [42]. These 
phenomenological forces and effective Lagrangians usually depend on about 10 adjustable parameters that are fitted to reproduce relevant ground-state properties, such as binding energies and charge radii of a few nuclei.

A common trend of phenomenological interactions used in the mean-field approach is their simple mathematical structure. In the nonrelativistic mean-field models, the Skyrme interactions [43,44] are among those most widely used. Skyrme forces are zero-range interactions that do not require calculations of exchange contributions. These forces have been employed for describing ground-state properties of the atomic nucleus, low-energy excited states, fission and fusion barriers, nucleon-nucleus and heavy-ion potentials, etc. (see, e.g., Ref. [44]). The parameter set $\mathrm{SkM}^{*}$ [31] is the classical paradigm of such a model. It is known to yield charge densities in overall agreement with densities inferred from experiment [45]. SLy4 [32] is a more modern version of the Skyrme force that was calibrated with special care for the isospin sector and for predictions of neutron-rich matter that occurs, e.g., in neutron stars.

The RMF theory of hadrons has become another useful tool for the study of bulk and single-particle properties of nuclear matter and finite nuclei [38,46-48]. In the relativistic model, nucleons are treated as Dirac particles that interact by exchanging virtual mesons. The covariant theory automatically takes into account the spin-orbit force, the finite range, and the density dependence of the nuclear interaction. The no-sea approximation, which disregards effects from the Dirac sea of negative energy states, is adopted. The open parameters of the model are the meson coupling constants and some of the meson masses. After fitting them to binding energies, charge radii, and other well-known empirical data of a few selected nuclei, the covariant theory predicts average properties of spherical and deformed nuclei over the whole periodic table in very good agreement with experiment $[35,49,50]$.

The original Walecka Lagrangian [46] contained $\sigma, \omega$, and $\rho$ mesons without any meson self-interactions. It was able to predict the correct saturation point of nuclear matter, albeit with a very large incompressibility modulus. The model was refined with the introduction of $\sigma$-meson self-interactions [51]. A parametrization of this type is, e.g., the celebrated NL3 model [33]. These parameter sets properly describe the data about finite nuclei, but often display differences, at densities above the saturation point, with microscopic Dirac-BruecknerHartree-Fock calculations of the nuclear matter equation of state [52,53]. A better agreement with the latter calculations at densities up to two to three times the saturation density is achieved by incorporating a quartic vector meson selfinteraction in the effective Lagrangian. Another interesting addition is a mixed isoscalar-isovector coupling [54], which allows one to modulate the density dependence of the nuclear symmetry energy. The variation of this coupling leaves the binding energy and proton rms of a finite nucleus almost unaltered, but it considerably modifies the rms radius of the neutron distribution. A representative instance of this type of model is the FSUGold parameter set [34]. This set yields an equation of state that is considerably softer than in NL3 for both symmetric matter and neutron matter. Apart from the binding energies and charge radii of nuclei,
FSUGold delivers a satisfactory description of several modes of collective excitations having different neutron-to-proton ratios.

The Lagrangian density associated with the $G 2$ parameter set is inspired by effective field theory methods. It contains all couplings consistent with the underlying QCD symmetries up to the order considered in the expansion scheme [35,38]. In contrast to the majority of mean-field models, $G 2$ describes the low-energy electromagnetic structure of the nucleon within the theory by means of vector-meson dominance and derivative couplings to the photon, cf. Ref. [35] for details. As indicated above, this means that no additional calculations with external nucleon form factors are needed to obtain the charge density and that the electromagnetic effects of the protons and neutrons are included within the low-energy regime in a unified framework [35]. The $G 2$ set explains finite nuclei and nuclear matter with a commendable level of accuracy. It predicts a soft equation of state both around saturation and at high densities that is consistent with recent measurements of kaon production and flow of matter in energetic heavy-ion collisions as well as with observations of masses and radii of neutron stars [55].

Recent formulations of the RMF theory do not introduce mesonic self-interactions but make the coupling constants of the mesons density dependent, like in the DD-ME2 parameter set [36]. These models accurately describe the properties of finite nuclei and, in addition, the associated equation of state of nuclear and neutron matter at suprasaturation agrees with the trends of microscopic Dirac-Brueckner-HartreeFock calculations that start from the bare nucleon-nucleon interaction.

Pairing correlations need to be taken into account for the calculation of open-shell nuclei. We will describe them in both nonrelativistic and relativistic frames, through a modified BCS approach that takes into account the continuum by means of quasibound levels due to their centrifugal (neutrons) or centrifugal-plus-Coulomb barriers (protons) [56]. For the Skyrme models used in this work, the pairing correlations are introduced by using a zero-range density-dependent force whose parameters can be found in Ref. [32]. In the case of the covariant NL3, G2, and FSUGold models, we describe the pairing correlations by means of a constant matrix element fitted to reproduce the experimental binding energies of some selected isotopic and isotonic chains [56]. In the DD-ME2 parameter set a fixed gap is considered, determined from experimental odd-even mass differences [36].

\section{B. Description of electron scattering}

The ELSEPA code [30] was originally designed for the calculation of elastic scattering of electrons and positrons by atoms, positive ions, and molecules. We have adapted it to handle high-energy electron scattering by nuclei. ELSEPA computes the DCS using the conventional relativistic partialwave method, which was first formulated by Yennie et al. [29]. The projectile electron is assumed to feel the electrostatic field of the nuclear charge distribution. The potential energy of an electron at a distance $r$ from the center of the nucleus is given 
by

$$
V(r)=-4 \pi e\left[\frac{1}{r} \int_{0}^{r} \rho_{\mathrm{ch}}\left(r^{\prime}\right) r^{\prime 2} d r^{\prime}+\int_{r}^{\infty} \rho_{\mathrm{ch}}\left(r^{\prime}\right) r^{\prime} d r^{\prime}\right]
$$

where $\rho_{\mathrm{ch}}(r)$ denotes the charge density of the nucleus, considered to be spherically symmetrical. At the energies of interest for the electron-nucleus problem, the effect of screening by the orbiting atomic electrons is limited to scattering angles smaller than $1^{\circ}$ (see, e.g. Ref. [30]), which are well below the angular range covered by the electron-nucleus scattering measurements. Consequently, electron screening is ignored in the present calculation. Because the nuclear charge density is assumed to vanish beyond a certain radius $r_{B}$ (i.e., the radius of the box where the nuclear charge distribution is calculated), the potential (2) is purely Coulombian, $V(r)=-Z e^{2} / r$, beyond that radius. Globally it can be regarded as a Coulomb potential with the short-range distortion arising from the finite size of the nucleus, i.e., as a modified Coulomb potential.

The DCS for elastic scattering of spin unpolarized electrons is given by

$$
\frac{d \sigma}{d \Omega}=|f(\theta)|^{2}+|g(\theta)|^{2}
$$

where

$$
\begin{aligned}
f(\theta)= & \frac{1}{2 \mathrm{i} k} \sum_{\ell=0}^{\infty}\left\{(\ell+1)\left[\exp \left(2 \mathrm{i} \delta_{\kappa=-\ell-1}\right)-1\right]\right. \\
& \left.+\ell\left[\exp \left(2 \mathrm{i} \delta_{\kappa=\ell}\right)-1\right]\right\} P_{\ell}(\cos \theta)
\end{aligned}
$$

and

$$
\begin{aligned}
g(\theta)= & \frac{1}{2 \mathrm{i} k} \sum_{\ell=0}^{\infty}\left[\exp \left(2 \mathrm{i} \delta_{\kappa=\ell}\right)\right. \\
& \left.-\exp \left(2 \mathrm{i} \delta_{\kappa=-\ell-1}\right)\right] P_{\ell}^{1}(\cos \theta)
\end{aligned}
$$

are the direct and spin-flip scattering amplitudes, respectively. Here $k$ denotes the wave number of the projectile electron,

$$
c \hbar k=\sqrt{E\left(E+2 m_{\mathrm{e}} c^{2}\right)},
$$

and the functions $P_{\ell}(\cos \theta)$ and $P_{\ell}^{1}(\cos \theta)$ are Legendre polynomials and associated Legendre functions, respectively. The phase shifts $\delta_{\kappa}$ represent the behavior of the Dirac spherical waves at large $r$ distances (see, e.g., Ref. [57]).

For modified Coulomb potentials, the spherical solutions of the Dirac equation are suitably expressed in the form

$$
\psi_{E \kappa m}(\mathbf{r})=\frac{1}{r}\left[\begin{array}{c}
P_{E \kappa}(r) \Omega_{\kappa, m}(\hat{\mathbf{r}}) \\
i Q_{E \kappa}(r) \Omega_{-\kappa, m}(\hat{\mathbf{r}})
\end{array}\right] .
$$

The functions $\Omega_{\kappa, m}(\hat{\mathbf{r}})$ are the spherical spinors, and the radial functions $P_{E \kappa}(r)$ and $Q_{E \kappa}(r)$ satisfy the following system of coupled differential equations [57]:

$$
\begin{aligned}
\frac{d P_{E \kappa}}{d r} & =-\frac{\kappa}{r} P_{E \kappa}+\frac{E-V+2 m_{\mathrm{e}} c^{2}}{c \hbar} Q_{E \kappa}, \\
\frac{d Q_{E \kappa}}{d r} & =-\frac{E-V}{c \hbar} P_{E \kappa}+\frac{\kappa}{r} Q_{E \kappa} .
\end{aligned}
$$

The relativistic quantum number $\kappa$ is defined as $\kappa=(\ell-j)$ $(2 j+1)$, where $j$ and $\ell$ are the total and orbital angular momentum quantum numbers. Note that $j$ and $\ell$ are both determined by the value of $\kappa ; j=|\kappa|-1 / 2, \ell=j+\kappa /(2|\kappa|)$. In the numerical calculations, the spherical waves are normalized so that the upper-component radial function $P_{E \kappa}(r)$ oscillates asymptotically with unit amplitude.

For modified Coulomb potentials and $r \rightarrow \infty$, we have (see, e.g., Ref. [58])

$$
P_{E \kappa}(r) \simeq \sin \left(k r-\ell \frac{\pi}{2}-\eta \ln 2 k r+\delta_{\kappa}\right),
$$

where

$$
\eta=Z e^{2} m_{\mathrm{e}} /\left(\hbar^{2} k\right)
$$

is the Sommerfeld parameter. It is convenient to express the phase shifts $\delta_{\kappa}$ as $\Delta_{\kappa}+\hat{\delta}_{\kappa}$, where $\Delta_{\kappa}$ is the phase shift of the point-nucleus Coulomb potential and $\hat{\delta}_{\kappa}$ is the "inner" phase shift of the short-range potential induced by the nuclear charge distribution.

As indicated above, the calculations reported here have been performed using the computer code ELSEPA [30]. It solves the radial Dirac equations using a robust integration algorithm, described in Refs. [58,59], which effectively minimizes the effect of truncation errors. The algorithm starts from a table of values of the function $r V(r)$ at the points $r_{i}$ of a radial grid, which is provided by the user. This function is replaced by the natural cubic spline that interpolates the tabulated values; thus, in the interval between consecutive grid points, the potential function $r V(r)$ is represented as a cubic polynomial. The radial wave equations (8) are then solved by using the exact power-series expansions of the radial functions [59]. The integration is started at $r=0$ and extended outwards up to a point $r_{m}$ that is beyond the starting radius $r_{B}$ of the Coulomb tail. For $r>r_{m}$, the field is purely Coulombian, and the normalized upper-component radial Dirac function can be expressed as

$$
P_{E \kappa}(r)=\cos \hat{\delta}_{\kappa} f_{E \kappa}^{(\mathrm{u})}(r)+\sin \hat{\delta}_{\kappa} g_{E \kappa}^{(\mathrm{u})}(r),
$$

where $f_{E \kappa}^{(\mathrm{u})}(r)$ and $g_{E \kappa}^{(\mathrm{u})}(r)$ are the upper components of the regular and irregular Dirac-Coulomb radial functions [58], respectively. As usual, the phase shift $\hat{\delta}_{\kappa}$ is determined by matching this outer analytical form to the inner numerical solution at $r_{m}$, requiring continuity of the radial function $P_{E \kappa}(r)$ and its derivative. The Dirac-Coulomb functions are calculated by using the Fortran subroutine described in Ref. [58], which delivers values of the regular and irregular Dirac-Coulomb functions and their derivatives that are accurate to more than 10 decimal figures.

The convergence rate of the series (4) and (5) for the calculation of $d \sigma / d \Omega$ is known to be slow. The summations are optimized by performing them in two steps [30]. First, they are evaluated for the pure Coulomb field, for which the phase shifts $\Delta_{\kappa}$ are known analytically and the calculation is fast. Second, the point-nucleus results are subtracted from the expansions (4) and (5); the remaining series represent the effect of only the short-range component of the potential and converge more rapidly than the original series. As a consequence, the number of inner phase shifts $\hat{\delta}_{\kappa}$ one needs to compute is normally much smaller than the number of required Coulomb phase shifts. 
In general, ELSEPA gives reliable results for electron energies up to $1 \mathrm{GeV}$ but numerical difficulties can appear at very small scattering angles because the DCS for the bare nucleus is very close to the point nucleus DCS (which is known as the Mott DCS) and consequently diverges. The code evaluates the finite-nucleus DCS only for scattering angles larger than 10 degrees, which is a lower bound of the usually measured electron-nucleus DCS. Depending on the particulars of each calculation, difficulties can also be found in the high-energy regime and for large scattering angles where the DCS takes much smaller values than the Mott DCS. In this case, the nuclear amplitudes almost cancel the Coulomb scattering amplitudes, thus magnifying the numerical errors. In practice, round-off errors become apparent when the nuclear scattering amplitudes are less then $10^{-5}$ times the Coulomb amplitudes (which are themselves small). When there are indications that these errors could be important, ELSEPA discontinues the calculation and the DCS is set to zero.

\section{ELASTIC ELECTRON-NUCLEUS SCATTERING IN STABLE NUCLEI}

As stated in the Introduction, we compute the chargedensity distributions with selected effective nuclear interactions, namely the Skyrme forces SkM* [31] and SLy4 [32] and the covariant parametrizations NL3 [33], FSUGold [34], G2 [35], and DD-ME2 [36]. We obtain the DCS from these meanfield charge densities using the ELSEPA code. Specifically, in the present section we compare the theoretical DCS derived from the mean-field models with available experimental data for elastic electron scattering in ${ }^{16} \mathrm{O}$ at $374.5 \mathrm{MeV}$ [60], ${ }^{40,42,44,48} \mathrm{Ca}$ and ${ }^{48} \mathrm{Ti}$ at $250 \mathrm{MeV},{ }^{40,48} \mathrm{Ca}$ at $500 \mathrm{MeV}$ [61], ${ }^{90} \mathrm{Zr}$ at 209.6 and $302 \mathrm{MeV}[62],{ }^{116,118,124} \mathrm{Sn}$ at $225 \mathrm{MeV}$ [63], and ${ }^{208} \mathrm{~Pb}$ at 248.2 and $502 \mathrm{MeV}$ [64].

In the conventional analyses of the scattering data measured in experiment, the charge density is usually modeled by means of an analytical function. For instance, two- or threeparameter Fermi distributions are used to this end. The charge density may also be constructed from the eigenfunctions of an adjustable single-particle potential of Woods-Saxon or harmonic oscillator type. Also, nearly model-independent charge densities are obtained from a Fourier-Bessel expansion [65] with unknown coefficients. In all these cases the free parameters in the charge distributions are determined from the measured electron scattering data through a least-squares minimization procedure. In some comparisons with our theoretical predictions we will employ experimental charge densities borrowed from the literature. These densities have been extracted from fits with Fourier-Bessel expansions [6,7]. In the case of the nucleus ${ }^{118} \mathrm{Sn}$, for which this type of density is not available, we employ the two-parameter Fermi distribution given in Ref. [63]. The results computed with these "experimental" (fitted) charge densities will be referred to as $\operatorname{Exp}$ (fit) in the tables and figures henceforth.

First, we report in Table I the rms radii of the theoretical charge distributions of the nuclei ${ }^{16} \mathrm{O},{ }^{40} \mathrm{Ca},{ }^{48} \mathrm{Ca},{ }^{90} \mathrm{Zr},{ }^{118} \mathrm{Sn}$, and ${ }^{208} \mathrm{~Pb}$ predicted by the Skyrme and RMF forces used in this work. They are compared with the rms radii of the experimental charge distributions extracted from the analysis of the electron
TABLE I. Root-mean-square radii (in $\mathrm{fm}$ ) for the studied charge distributions. $\operatorname{Exp}($ fit) values are calculated from the experimental Fourier-Bessel charge densities [6,7], except for the case of ${ }^{118} \mathrm{Sn}$ where a Fermi density is used [63].

\begin{tabular}{lccccccc}
\hline \hline Nucleus & Exp(fit) & DD-ME2 & G2 & NL3 & FSUGold & SLy4 & SkM $^{*}$ \\
\hline${ }^{16} \mathrm{O}$ & 2.74 & 2.73 & 2.73 & 2.73 & 2.69 & 2.80 & 2.81 \\
${ }^{40} \mathrm{Ca}$ & 3.45 & 3.46 & 3.46 & 3.47 & 3.44 & 3.51 & 3.52 \\
${ }^{48} \mathrm{Ca}$ & 3.45 & 3.48 & 3.45 & 3.47 & 3.47 & 3.54 & 3.54 \\
${ }^{90} \mathrm{Zr}$ & 4.26 & 4.28 & 4.25 & 4.28 & 4.26 & 4.30 & 4.29 \\
${ }^{118} \mathrm{Sn}$ & 4.67 & 4.63 & 4.62 & 4.63 & 4.63 & 4.65 & 4.63 \\
${ }^{208} \mathrm{~Pb}$ & 5.50 & 5.52 & 5.50 & 5.52 & 5.52 & 5.52 & 5.51 \\
\hline \hline
\end{tabular}

scattering data $[6,7,63]$. The agreement is seen to be very good. This fact is not surprising because some of the experimental values of the charge radius have been used in the fit of the free parameters of the Skyrme forces and RMF models considered.

Figure 1 displays the theoretical charge density profiles obtained with the investigated mean-field models as well as the experimental charge distributions for ${ }^{16} \mathrm{O},{ }^{90} \mathrm{Zr}$, and ${ }^{208} \mathrm{~Pb}[6,7]$. In general, theoretical and experimental charge densities agree nicely in the fall-off region and differ more in the nuclear interior as a consequence of the shell oscillations of the mean-field densities. Differences in the inner region are more marked in light nuclei where a mean-field approximation

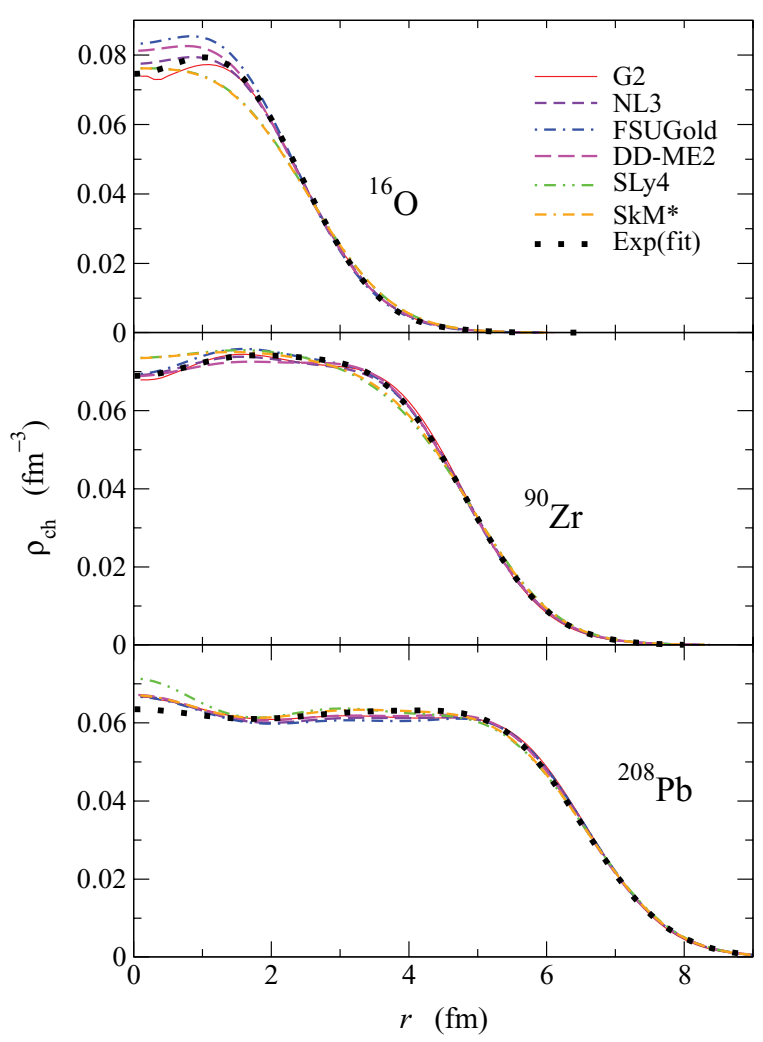

FIG. 1. (Color online) Radial dependence of the charge densities of the stable nuclei ${ }^{16} \mathrm{O},{ }^{90} \mathrm{Zr}$, and ${ }^{208} \mathrm{~Pb}$. The predictions of the different mean-field models indicated in the legend are compared with the charge densities fitted experimentally by the Fourier-Bessel analysis [6,7]. 
may be less justified. In the present case, if we analyze the region of the experimental density of ${ }^{16} \mathrm{O}$ between the center and about $2 \mathrm{fm}$, we see that the covariant sets $G 2$ and NL3 give a better description, whereas the $\mathrm{SkM}^{*}$ and SLy4 forces tend to underestimate it and the DD-ME2 and FSUGold sets tend to overestimate it. A detailed inspection of the mean-field charge densities shows that they differ not only among themselves in the nuclear interior but also in the surface region. As discussed in Ref. [45], the inner density is normally larger for nuclei with larger surface diffuseness. From Fig. 1 we see that for medium and heavy nuclei the Skyrme charge density, in particular the one predicted by SLy4, is larger in the interior and consequently more diffuse at the surface than the RMF distributions. Differences regarding the surface diffuseness between non-relativistic and relativistic charge densities are related to the different density dependence of the effective interactions [45].

The comparison between theoretical and experimental charge densities shall be connected with the discussion of the DCS and the electric charge form factors, which are the quantities measured in real experiments. Figure 2 shows the DCS for elastic electron scattering in ${ }^{16} \mathrm{O}$ at $374.5 \mathrm{MeV}$, ${ }^{90} \mathrm{Zr}$ at $302 \mathrm{MeV}$, and ${ }^{208} \mathrm{~Pb}$ at $502 \mathrm{MeV}$, which we choose as representative examples to illustrate the predictions of the different mean-field models. To improve readability, the curves calculated with NL3 and $\mathrm{SkM}^{*}$ are not displayed in the figure. In the three scattering processes, one observes that the experimental data are similarly reproduced by all of the considered mean-field charge densities at small scattering

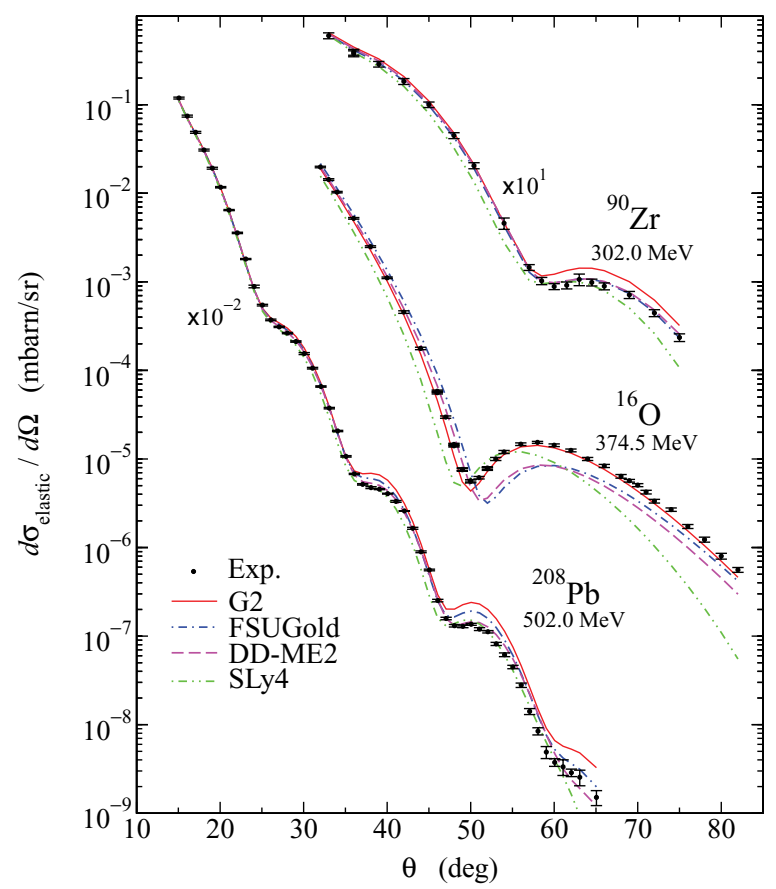

FIG. 2. (Color online) Elastic DCS for electron-nucleus scattering in ${ }^{16} \mathrm{O},{ }^{90} \mathrm{Zr}$, and ${ }^{208} \mathrm{~Pb}$ as a function of the scattering angle $\theta$, at the energies shown. The results from the mean-field models indicated in the legend are compared with the measured DCS (Exp) [60-62] and with the DCS calculated in the ELSEPA code from the charge densities fitted experimentally by the Fourier-Bessel analysis [Exp(fit)] [6,7]. angles, up to the first diffraction minimum. The statement is more valid for lead, where models and data keep close up to the second, or even third, diffraction minimum. The case which is seen to pose more difficulties to the mean-field models at all scattering angles is, not surprisingly, the lightest investigated nucleus, ${ }^{16} \mathrm{O}$. From the considered interactions, only the parameter set $G 2$ is able to reproduce the experimental data of ${ }^{16} \mathrm{O}$ at $374.5 \mathrm{MeV}$ closely at all scattering angles. As one might expect from the discussion of Fig. 1, the deviations among the DCS predicted by the various mean-field models, and the discrepancies with respect to experiment, become more prominent at the largest scattering angles in the three processes studied in Fig. 2. One detects a significant difference between the nonrelativistic Skyrme interactions and the RMF models used here. It is seen that the SLy4 interaction yields DCS values that, in general, are smaller than those calculated using the charge densities of the covariant sets (the same happens with the Skyrme force $\mathrm{SkM}^{*}$ ). This trend is especially clear in ${ }^{16} \mathrm{O}$ and ${ }^{90} \mathrm{Zr}$, if one just excludes the region immediately after the first diffraction minimum where a crossing of the DCS values predicted by some of the models takes place.

One of the most effective quantities to characterize elastic electron-nucleus scattering is the electric charge form factor $F(q)$. The momentum transfer $q$ is related to the scattering angle $\theta$ in the laboratory frame by

$$
c \hbar q=2 E \sin (\theta / 2) \text {. }
$$

In PWBA the electric-charge form factor is computed as the Fourier transform of the charge density. In the present work

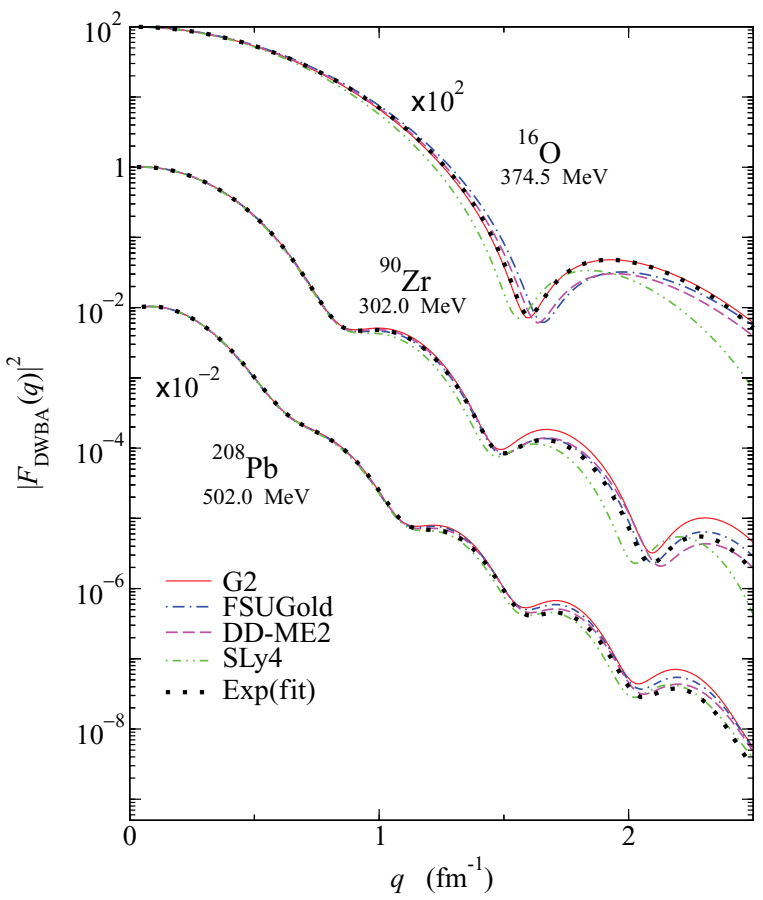

FIG. 3. (Color online) Squared charge form factor for ${ }^{16} \mathrm{O},{ }^{90} \mathrm{Zr}$, and ${ }^{208} \mathrm{~Pb}$ as a function of the momentum transfer $q$ at the electron beam energies shown. It has been obtained by applying Eq. (13) as described in the text, both for the mean-field models indicated in the legend and for the experimentally fitted charge densities [Exp(fit)] $[6,7]$. 

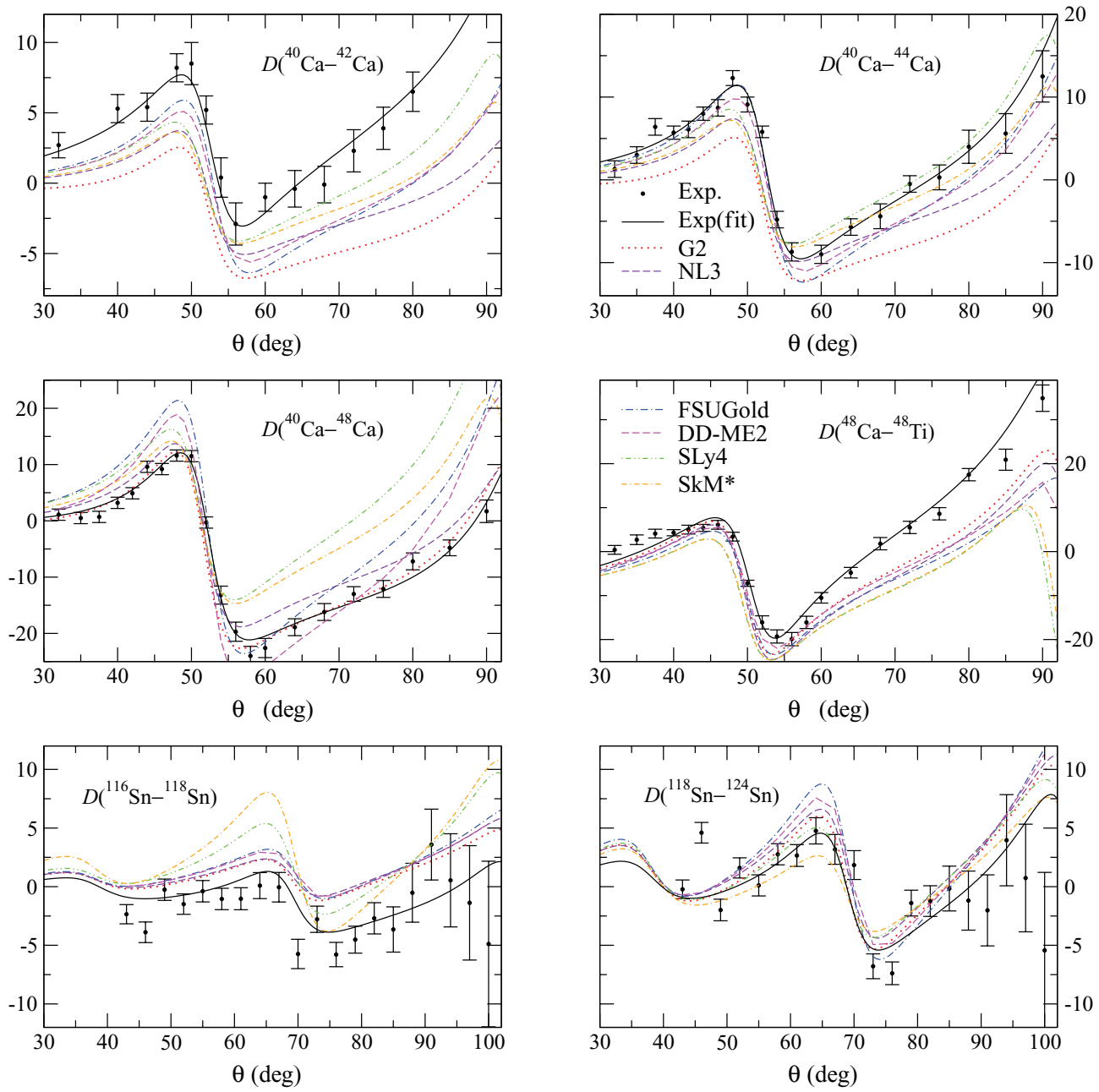

FIG. 4. (Color online) Relative differences of DCS in pairs of neighbor nuclei at $250 \mathrm{MeV}$ for $\mathrm{Ca}$ and $\mathrm{Ti}$ and at $225 \mathrm{MeV}$ for $\mathrm{Sn}$. The results from mean-field models are compared with the measured RDDCS (Exp) and with the RDDCS calculated in the ELSEPA code from the charge densities fitted experimentally [Exp(fit)] in Refs. [61,63]. Note that in the present figure the vertical scales are linear and that all of them have been magnified by a factor 100 .

we obtain $|F(q)|^{2}$ at a given beam energy as

$$
|F(q)|^{2}=\left(\frac{d \sigma}{d \Omega}\right)\left(\frac{d \sigma_{M}}{d \Omega}\right)^{-1}
$$

where $d \sigma / d \Omega$ is the DCS calculated from the DWBA analysis and $d \sigma_{M} / d \Omega$ is the Mott DCS. Equation (13) goes beyond the first Born approximation to the charge form factor because, instead of the PWBA for the DCS of the point nucleus, the exact Mott DCS is used. It will be referred to as $F_{\mathrm{DwBA}}(q)$ in what follows. We will extract the squared charge form factor from experiment using the same expression (13), by inserting the DCS obtained from the experimentally fitted charge densities on its right-hand side.

Figure 3 displays the $q$ dependence of $\left|F_{\mathrm{DWBA}}(q)\right|^{2}$ for the nuclei ${ }^{16} \mathrm{O},{ }^{90} \mathrm{Zr}$, and ${ }^{208} \mathrm{~Pb}$ at the electron beam energies 374.5 , 302 , and $502 \mathrm{MeV}$, respectively. Results are calculated from the considered mean-field models and from the experimental charge density (as in Fig. 2, the curves obtained with NL3 and $\mathrm{SkM}^{*}$ are not shown). As it may be expected from our previous analysis of the DCS, the experimental values of $|F(q)|^{2}$ are well reproduced in the low-momentum transfer region by all of the discussed mean fields. However, some discrepancies appear between the theoretical predictions and the experimental data at large-momentum transfers (or, equivalently, at large scattering angles). They are an indication that the theoretical mean-field models describe differently the central region of the experimental charge density [60], which we have addressed in the discussions of Fig. 1.

A more exigent test of the mean-field charge densities than the DCS themselves is provided by the analysis of the relative differences of DCS values between pairs of neighbor nuclei (RDDCS). The RDDCS are defined as

$$
D(A-B)=\frac{(d \sigma / d \Omega)_{A}-(d \sigma / d \Omega)_{B}}{(d \sigma / d \Omega)_{A}+(d \sigma / d \Omega)_{B}} .
$$

We explore the following cases where experimental values are available: ${ }^{40} \mathrm{Ca}^{-}{ }^{42} \mathrm{Ca},{ }^{40} \mathrm{Ca}-{ }^{44} \mathrm{Ca},{ }^{40} \mathrm{Ca}-{ }^{48} \mathrm{Ca}$, and ${ }^{48} \mathrm{Ca}$ ${ }^{48} \mathrm{Ti}$ [61], as well as ${ }^{116} \mathrm{Sn}-{ }^{118} \mathrm{Sn}$ and ${ }^{118} \mathrm{Sn}-{ }^{124} \mathrm{Sn}$ [63]. The theoretical predictions for the RDDCS (14) from the meanfield charge densities are displayed against experiment in Fig. 4, where a factor of 100 has been introduced for the sake of clarity. Notice that the vertical scale of this figure is 
TABLE II. Normalized square weighted difference $\left(d_{w}^{2}\right)$ between the calculated and measured DCS values. $\operatorname{Exp}$ (fit) values are calculated from the experimental Fourier-Bessel charge densities [6,7], except for the case of ${ }^{118} \mathrm{Sn}$ where a Fermi density is used [63]. The energy $E$ of the incident electrons is in $\mathrm{MeV}$.

\begin{tabular}{llccccccc}
\hline \hline Nucleus & $E$ & Exp(fit) & DD-ME2 & $G 2$ & NL3 & FSUGold & SLy4 & SkM $^{*}$ \\
\hline${ }^{16} \mathrm{O}$ & 374.5 & 11.1 & 88.7 & 13.1 & 38.6 & 206 & 191 & 194 \\
${ }^{40} \mathrm{Ca}$ & 250 & 7.18 & 3.15 & 16.2 & 13.9 & 0.84 & 24.4 & 24.3 \\
& 500 & 3.48 & 1.49 & 42.9 & 19.7 & 5.79 & 40 & 39 \\
${ }^{48} \mathrm{Ca}$ & 250 & 6.66 & 4.85 & 9.74 & 7.14 & 4.08 & 14.9 & 13.6 \\
& 500 & 3.19 & 1.11 & 17 & 3.53 & 2.57 & 21.84 & 18.5 \\
${ }^{90} \mathrm{Zr}$ & 209.6 & 0.78 & 0.87 & 2.21 & 1.36 & 0.65 & 6.53 & 5.36 \\
& 302 & 0.86 & 0.91 & 9.92 & 3.27 & 0.67 & 9.35 & 7.19 \\
${ }^{118} \mathrm{Sn}$ & 225 & 5.43 & 18.4 & 34.8 & 25.5 & 31.8 & 2.75 & 4.2 \\
${ }^{208} \mathrm{~Pb}$ & 248.2 & 30.6 & 44.4 & 154 & 74.8 & 89.5 & 89.2 & 61 \\
& 502 & 21.2 & 14.1 & 186 & 50.5 & 61.1 & 95.9 & 76.5 \\
\hline \hline
\end{tabular}

linear instead of logarithmic. In general, all the considered models describe fairly well the experimental RDDCS values for small scattering angles. The agreement with experiment deteriorates when the scattering angle increases, pointing out again some possible deficiencies in the inner region of the theoretical charge density distributions.

To make a more quantitative analysis of the electron scattering DCS derived from the mean-field charge densities, we introduce a normalized square weighted difference $\left(d_{w}^{2}\right)$, or comparison magnitude, with respect to the DCS measured in experiment. For each nucleus and electron beam energy, it is defined as

$$
d_{w}^{2}=\frac{1}{N} \sum_{i=1}^{N}\left[\frac{(d \sigma / d \Omega)_{i}^{\exp }-(d \sigma / d \Omega)_{i}^{\text {calc }}}{\delta(d \sigma / d \Omega)_{i}^{\exp }}\right]^{2} .
$$

In this expression, the quantities $(d \sigma / d \Omega)_{i}^{\text {calc }},(d \sigma / d \Omega)_{i}^{\exp }$, and $\delta(d \sigma / d \Omega)_{i}^{\exp }$ are, respectively, the calculated DCS, the measured DCS, and the uncertainty of the latter. The sum in Eq. (15) runs over all the $N$ available data for the given scattering process.

In Table II we report, for several elastic electron-nucleus reactions, the $d_{w}^{2}$ values (15) from theory in comparison with the $d_{w}^{2}$ obtained from the charge densities fitted experimentally. One realizes that the $d_{w}^{2}$ of the DCS computed with the theoretical charge densities take sizably varying values, depending on the scattering process and nuclear model. Nevertheless, for all the considered electron-scattering processes, there are Skyrme forces or RMF parametrizations that yield a $d_{w}^{2}$ of similar quality to the experimental charge density. In particular, the RMF sets DD-ME2 and FSUGold give, on the average, the best overall agreement with the considered experimental data. The Skyrme forces $\mathrm{SkM}^{*}$ and SLy4 provide a similar description, but globally this description is slightly worse than in the case of the RMF parametrizations, except for the nucleus ${ }^{118} \mathrm{Sn}$.

We have also analyzed the $d_{w}^{2}$ values of the relative differences between differential cross sections (14) in pairs of neighbor nuclei. The corresponding results are collected in Table III. For all the mean fields considered in the present study, the predicted $d_{w}^{2}$ values are larger than the $d_{w}^{2}$ values computed with the charge densities that are fitted to experiment. Looking globally at the results presented in Table III, the agreement obtained between theoretical and experimental RDDCS is not significantly worse than the agreement found in the case of the DCS analyzed previously in Table II. But, in the DCS analysis, it has been seen in Table II that for each scattering process there is always one or various specific interactions that are able to come very close to experiment, whereas in Table III this does not happen in any of the studied RDDCS in pairs of neighbor nuclei.

\section{ELECTRON SCATTERING ALONG ISOTOPIC CHAINS}

Different tendencies of the square of the charge form factor as a function of the momentum transfer have been

TABLE III. Normalized square weighted difference $\left(d_{w}^{2}\right)$ between calculated and measured relative differences of DCS in pairs of neighbor nuclei. The beam energy per electron is $250 \mathrm{MeV}$ for the $\mathrm{Ca}$ isotopes and ${ }^{48} \mathrm{Ti}$, and $225 \mathrm{MeV}$ for the $\mathrm{Sn}$ isotopes.

\begin{tabular}{lccccccc}
\hline \hline$D(A-B)$ & Exp(fit) & DD-ME2 & $G 2$ & NL3 & FSUGold & SLy4 & SkM $^{*}$ \\
\hline$D\left({ }^{40} \mathrm{Ca}-{ }^{42} \mathrm{Ca}\right)$ & 0.56 & 9.1 & 28.3 & 16 & 11.1 & 9.11 & 12.9 \\
$D\left({ }^{40} \mathrm{Ca}-{ }^{44} \mathrm{Ca}\right)$ & 1.14 & 4.5 & 29.6 & 12.2 & 3.88 & 7.08 & 9.13 \\
$D\left({ }^{40} \mathrm{Ca}-{ }^{48} \mathrm{Ca}\right)$ & 1.06 & 16.4 & 4.89 & 7.74 & 38.5 & 94.1 & 49.3 \\
$D\left({ }^{48} \mathrm{Ca}-{ }^{48} \mathrm{Ti}\right)$ & 2.49 & 18 & 19.6 & 31 & 37.8 & 71.8 & 64.9 \\
$D\left({ }^{116} \mathrm{Sn}-{ }^{118} \mathrm{Sn}\right)$ & 2.05 & 8.05 & 7.8 & 9 & 10.1 & 13.2 & 18.5 \\
$D\left({ }^{118} \mathrm{Sn}-{ }^{124} \mathrm{Sn}\right)$ & 4.03 & 5.35 & 6.98 & 7.5 & 9.22 & 7.05 & 7.18 \\
\hline \hline
\end{tabular}


studied in earlier literature for isotopic $[20,21,25]$ and isotonic [26] chains of medium and heavy nuclei. Our aim here is to perform an analysis, quantitative whenever possible, that could eventually be useful for future electron-scattering measurements in RIB facilities.

We will be concerned with the study of electron scattering in isotopic chains of medium and relatively heavy systems, which will be exemplified by the cases of the $\mathrm{Ca}$ and $\mathrm{Sn}$ isotopes. Many of these nuclei lie in the region of the nuclear chart that is likely to be explored employing RIB facilities. Some of them may be investigated in future electron-scattering experiments, such as ELISe [17] and SCRIT [18,19]. For the purpose of our study, we are interested in predicting the trends of the variation along the isotopic chains of the electric charge form factor in the low-momentum transfer regime. Our calculated mean-field charge densities will be parameterized by means of the Helm model [66], often used in the analysis of experimental data, with a view to gain deeper physical insights and to elucidate possible correlations among scattering observables within the isotopic chains.

We have seen in the previous section that in the region of small $q$ values the experimental results are almost equally well reproduced by the calculations with the different theoretical mean-field models considered. In our subsequent study of electron scattering in the tin and calcium chains, as a representative reference, we will work with the charge densities predicted by the covariant interaction $G 2[35,38]$. This model was constructed as an effective hadronic Lagrangian consistent with the symmetries of quantum chromodynamics. As mentioned, the model describes the low-energy electromagnetic structure of the nucleon using vector-meson dominance and provides directly the charge density of the nucleus so no external single-nucleon form factors are required to compute the latter [35]. $G 2$ is also a reliable parameter set for calculations of ground states of nuclei and, at the same time, for predictions of the nuclear equation of state up to supranormal densities and of some properties of neutron stars [35,38,55]. Calculations of the squared charge form factor done in PWBA with the set $G 2$ for stable isotopes have been reported elsewhere [35].

For each nucleus in an isotopic chain we compute the associated DCS via the DWBA calculation using the $G 2$ charge density. The electron beam energy in all the investigated scattering processes is fixed at $500 \mathrm{MeV}$. In practice, the energy dependence of the electric charge form factor defined in Eq. (13) is seen to be considerably weak for low-momentum transfers, the regime addressed in our analysis. We have verified numerically that $\left|F_{\mathrm{DWBA}}(q)\right|^{2}$ of high-energy electron scattering depends little on the electron beam energy for momentum transfers up to $1-1.5 \mathrm{fm}^{-1}$ (the precise value depends on the nucleus). This happens even for a heavy system like ${ }^{208} \mathrm{~Pb}$, where the departure from the point-nucleus assumption of the Mott DCS used on the right-hand side of Eq. (13) is more significant. We illustrate the situation in Fig. 5, where we display $\left|F_{\text {DWBA }}(q)\right|^{2}$ at several beam energies for electron scattering off ${ }^{118} \mathrm{Sn}$ and ${ }^{208} \mathrm{~Pb}$.

Before proceeding to the presentation of the results in the tin and calcium isotopic chains, we briefly summarize in the next subsection how we determine the parameters of the Helm model charge-density distributions.

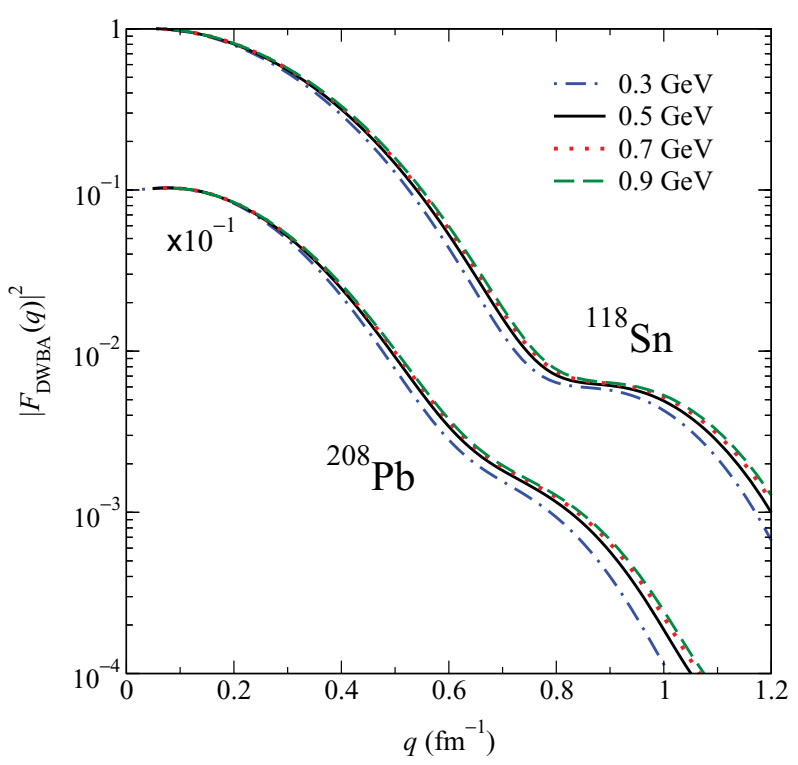

FIG. 5. (Color online) Squared charge form factor of ${ }^{118} \mathrm{Sn}$ and ${ }^{208} \mathrm{~Pb}$, derived from the covariant mean-field model $G 2$, for the indicated electron beam energies. The figure points out that the sensitivity of the charge form factor extracted through Eq. (13) to the beam energy in high-energy elastic electron-nucleus scattering is rather small, even when the atomic number of the target is large as in ${ }^{208} \mathrm{~Pb}$.

\section{A. Equivalent Helm charge densities}

Valuable insights into the study of electron scattering often stem from consideration of modeled charge densities and electric charge form factors. Moreover, these parameterized forms are instrumental in experimental data analyses. A notable case is the so-called Helm model, whose original version [66] has later been extended in various ways for more accurate descriptions of the experimental charge densities [67-69]. In the simpler version of the model, two chief features of the nuclear charge density, namely the position and the thickness of the surface, can be related explicitly to the electric charge form factor obtained in PWBA. The Helm charge density is obtained from the convolution of a constant density $\rho_{0}$ in a hard sphere of radius $R_{0}$ (the diffraction radius) with a Gaussian distribution of variance $\sigma^{2}$ (whose square root relates to the nuclear surface thickness):

$$
\rho^{(H)}(\vec{r})=\int d \vec{r}^{\prime} f_{G}\left(\vec{r}-\vec{r}^{\prime}\right) \rho_{0} \Theta\left(R_{0}-r\right),
$$

where

$$
f_{G}(r)=\left(2 \pi \sigma^{2}\right)^{-3 / 2} e^{-r^{2} / 2 \sigma^{2}}
$$

The rms radius of the Helm density is readily obtained from Eqs. (16) and (17). It can be expressed in terms of $R_{0}$ and $\sigma$ as

$$
\left\langle r^{2}\right\rangle_{H}^{1 / 2}=\sqrt{\frac{3}{5}\left(R_{0}^{2}+5 \sigma^{2}\right)} .
$$


The corresponding electric charge form factor in PWBA is given by

$$
F^{(H)}(q)=\int e^{i \vec{q} \cdot \vec{r}} \rho^{(H)}(\vec{r}) d \vec{r}=\frac{3}{q R_{0}} j_{1}\left(q R_{0}\right) e^{-\sigma^{2} q^{2} / 2},
$$

where $j_{1}(x)$ is a spherical Bessel function.

The diffraction radius $R_{0}$ of the Helm density is usually fixed as follows. One requires that the first zero of Eq. (19) occurs at $q_{\min } R_{0}$, where $q_{\min }$ corresponds to the first minimum of the modulus of the PWBA form factor $\left[F_{\mathrm{PWBA}}(q)\right.$ hereinafter] associated to the original charge distribution that the Helm density attempts to describe:

$$
R_{0}=\frac{4.49341}{q_{\min }} .
$$

The variance $\sigma^{2}$ of the Gaussian is chosen to reproduce the height of the second maximum of $\left|F_{\mathrm{PWBA}}(q)\right|$, located at $q_{\max }$ :

$$
\sigma^{2}=\frac{2}{q_{\max }^{2}} \ln \left[\frac{3 j_{1}\left(q_{\max } R_{0}\right)}{q_{\max } R_{0} F_{\mathrm{PWBA}}\left(q_{\max }\right)}\right] .
$$

For moderate values of $q R_{0}$, the Helm charge form factor $F^{(H)}(q)$ reproduces well the actual charge form factor, with the exception of the regions closest to its zeros [66]. The relative difference between $F^{(H)}(q)$ and the actual charge form factor becomes progressively manifest as the momentum transfer grows. The applicability of the Helm model near the drip lines is to be explored. The extent to which it may be appropriate away from stability, for the purposes of our study, will be validated later from the numerical point of view in connection with the discussion of the calculations in the $\mathrm{Sn}$ and Ca chains.

\section{B. Tin and calcium isotopic chains}

We are interested in the study of elastic electron scattering in the $\mathrm{Ca}$ and Sn chains. The calculated DCS and squared charge form factors $\left|F_{\text {DWBA }}(q)\right|^{2}$ show, for the lightest nuclei considered here (calcium isotopes), a relatively well-marked first minimum. This first minimum, however, practically disappears for the heavier nuclei analyzed (tin isotopes). (This fact can also be told from the previous Figs. 2 and 3 for ${ }^{16} \mathrm{O},{ }^{90} \mathrm{Zr}$, and ${ }^{208} \mathrm{~Pb}$.) In the latter case, the form factor $\left|F_{\mathrm{DWBA}}(q)\right|^{2}$ of Eq. (13) still shows an inflection point (IP) in the low-momentum transfer region, a point where the curvature changes sign. In the absence of an explicit minimum at low-momentum transfer, these IP are the best candidates to characterize along the isotopic chain relevant properties of the electric charge form factor in the small- $q$ region.

We determine for each isotope an equivalent Helm distribution from the calculated mean-field charge density. In the PWBA the distortion of the electron wave functions due to the Coulomb potential of the nucleus is neglected. The effect of the Coulomb attraction felt by the electrons can be simulated by replacing the momentum transfer $q$ by an effective value [66]

$$
q_{\mathrm{eff}}=q\left(1+c \frac{Z \alpha}{q R_{\mathrm{ch}}}\right),
$$
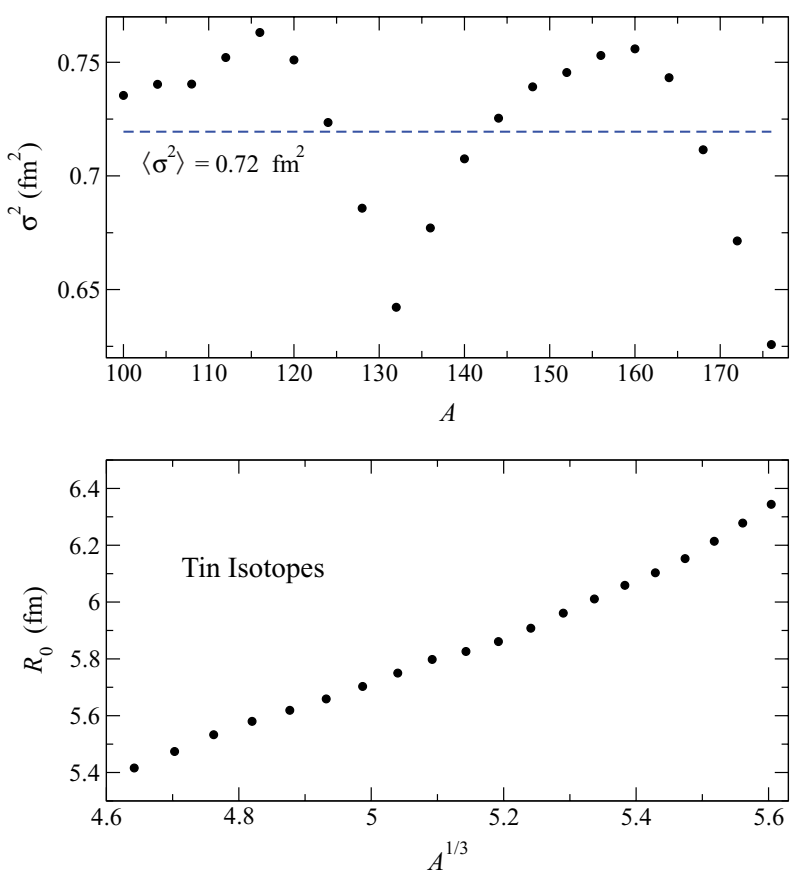

FIG. 6. (Color online) Mass-number dependence of the Helm parameters $\sigma^{2}$ (upper panel) and $R_{0}$ (lower panel) predicted by the covariant mean-field model $G 2$ in the $\mathrm{Sn}$ isotopic chain. The average value of $\sigma^{2}$ is depicted by a horizontal dashed line.

where $R_{\mathrm{ch}}=\sqrt{3 / 5} R$ is the rms of the charge density assuming a hard sphere distribution of radius $R=r_{0} A^{1 / 3}$. In Ref. [66] the value of the constant $c$ is taken to be $3 / 2$. Here we leave $c$ as a free parameter. It is optimized so that the rms radii of the equivalent Helm charge densities (18), with $R_{0}$ and $\sigma$ determined by Eqs. (20) and (21), and with $q$ replaced by $q_{\text {eff }}$, best reproduce along the isotopic chain the mean field rms charge radii obtained with the RMF parametrization $G 2$. Proceeding in this way, we find $c \approx 0.15$ for the tin isotopes and $c \approx 0.12$ for the calcium isotopes.

We first investigate the tin isotopic chain. The calculated Helm parameters $R_{0}$ and $\sigma^{2}$ are displayed in the lower and upper panels of Fig. 6, respectively. It is seen that $R_{0}$ steadily increases with the mass number $A$ and that it roughly follows the typical $A^{1 / 3}$ law. On the contrary, the change of the variance $\sigma^{2}$ with mass number shows a nonuniform character along the isotopic chain, related to the underlying shell structure. It oscillates around a mean value $\sigma^{2} \simeq 0.72(\sigma \simeq 0.85)$. It is to be noted that $\sigma^{2}$ displays local minima at the doubly magic isotopes ${ }^{132} \mathrm{Sn}$ and ${ }^{176} \mathrm{Sn}$ (neutron drip line nucleus). This fact points out a stiffer nuclear surface for neutron-rich nuclei with double-closed major shells, in agreement with earlier literature [67].

The upper panel of Fig. 7 depicts the radial dependence of the mean-field charge-density profiles computed with the $G 2$ covariant interaction for the isotopes ${ }^{100} \mathrm{Sn},{ }^{132} \mathrm{Sn}$, and ${ }^{176} \mathrm{Sn}$. We have selected these examples to illustrate the evolution of the results along the isotopic chain from one drip line to another. Though the three isotopes share the same atomic number, one notices outstanding variations in the calculated 

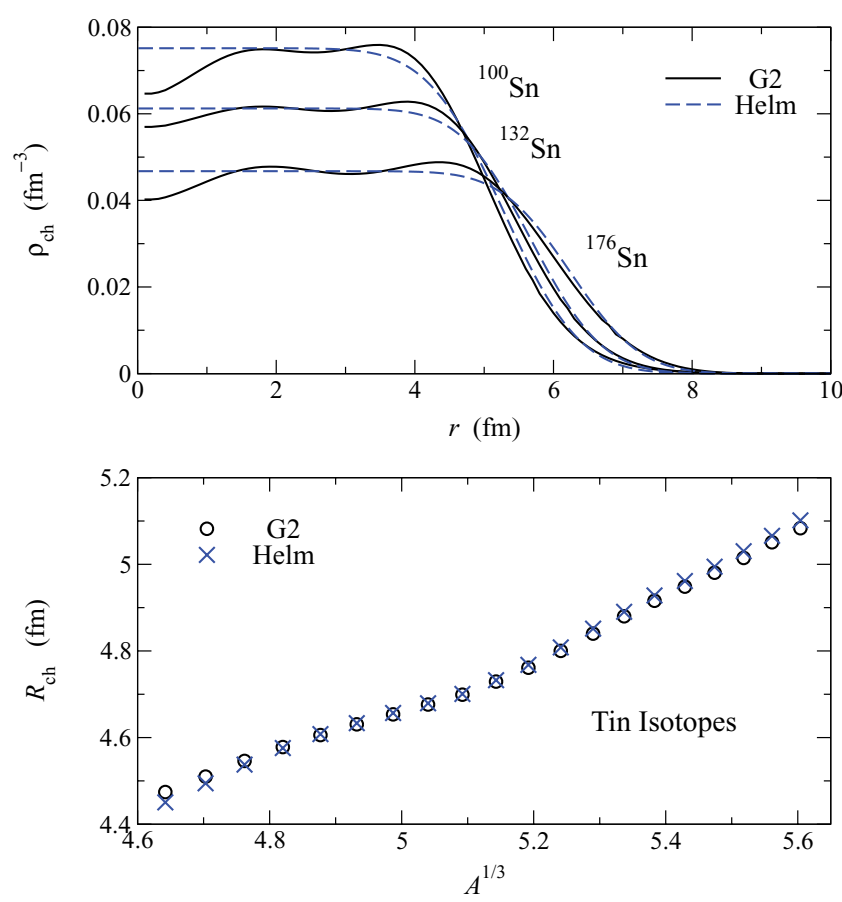

FIG. 7. (Color online) Charge densities (upper panel) and charge radii (lower panel) in the Sn isotopic chain, according to the covariant model $G 2$ and to the determined Helm distributions.

mean-field charge densities of these isotopes, both in the surface region and, especially, in the interior region. This fact reflects the important influence of the changing neutron number on driving the structure of the charge density along the isotopic chain. This influence is encoded in the interaction terms of the covariant Lagrangian of $G 2$ through the exchanged mesons and the couplings to the photon, and it is accounted for self-consistently by the mean-field calculations. In the same figure we compare the fitted Helm charge densities and the original $G 2$ charge densities for the three discussed tin isotopes. At the interior of these nuclei, we see that the uniform density of the Helm model averages the oscillations of the self-consistent quantal densities obtained with the $G 2$ interaction. In spite of the fact that the surface falloff of the Helm densities is of Gaussian type, the agreement at the surface region between the mean-field charge densities and their Helm equivalents is fairly good as one proceeds along the isotopic chain from stability to the neutron and proton drip lines. This provides some confidence on using the Helm model as determined in the present work when the drip lines are approached in the $\mathrm{Sn}$ chain. The lower panel of Fig. 7 illustrates the mass number dependence of the mean field and the equivalent Helm rms charge radii. Our approach leads to an excellent agreement between the Helm values and the mean-field values in the Sn chain. One appreciates some slight discrepancies only for isotopes very close to the drip lines. The rms charge radii of both calculations follow the expected linear trend with $A^{1 / 3}$. One observes some departure from the $A^{1 / 3}$ behavior to slightly lower values in the isotopes close to $A=132$. This is finally the reason why
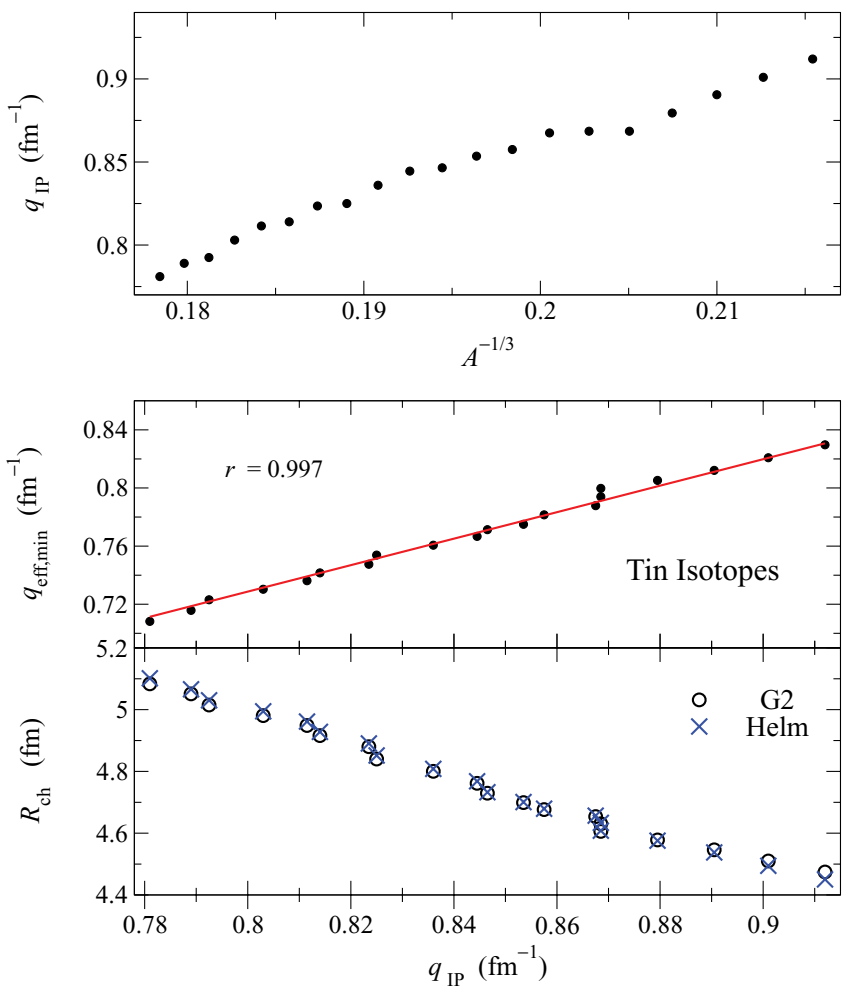

FIG. 8. (Color online) Results predicted by the $G 2$ effective interaction for $500-\mathrm{MeV}$ electron scattering in the Sn isotopic chain. (Upper panel) Mass-number dependence of the momentum transfer at the first inflection point $\left(q_{\mathrm{IP}}\right)$ of the squared charge form factor in DWBA. (Middle panel) Correlation of the effective momentum transfer at the first minimum of the squared charge form factor in PWBA $\left(q_{\text {eff,min }}\right)$ with the value of $q_{\mathrm{IP}}$. A linear fit of the results is shown and the correlation coefficient $r$ is indicated. (Lower panel) The change of the charge radii calculated with $G 2$ and with the corresponding Helm densities is depicted against the value of $q_{\mathrm{IP}}$.

the variance $\sigma^{2}$ decreases around $A=132$ in Fig. 6 [also see Eq. (18)].

We now analyze the evolution along the isotopic chain of the momentum transfer $q_{\mathrm{IP}}$ corresponding to the first inflection point of $\left|F_{\mathrm{DWBA}}(q)\right|^{2}$ and the variation of $\left|F_{\mathrm{DWBA}}\left(q_{\mathrm{IP}}\right)\right|^{2}$, calculated for an electron beam energy of $500 \mathrm{MeV}$. We discuss possible correlations with the parameters $R_{0}$ and $\sigma$ of the equivalent Helm density. Two noticeable findings of this study are displayed in Fig. 8. In the upper panel of the figure it is seen that the change of the momentum transfer $q_{\text {IP }}$ with mass number in the $\mathrm{Sn}$ isotopic chain approximately follows an $A^{-1 / 3}$ law. Thus, the position of the first minimum of the square of the modulus of the electric charge form factor is shifted toward smaller $q$ values as the number of neutrons in the isotopic chain increases, a feature also noted in Ref. [25]. Another interesting result is that the momentum transfer $q_{\mathrm{IP}}$ turns out to be almost proportional to the effective momentum transfer $q_{\text {eff }}$ corresponding to the corrected first minimum of $\left|F_{\mathrm{PWBA}}(q)\right|$ computed with the mean-field charge density. The situation is illustrated in the middle panel of Fig. 8. Recalling Eq. (20), this correlation allows one to establish a straightforward relationship between $q_{\mathrm{IP}}$ and the 


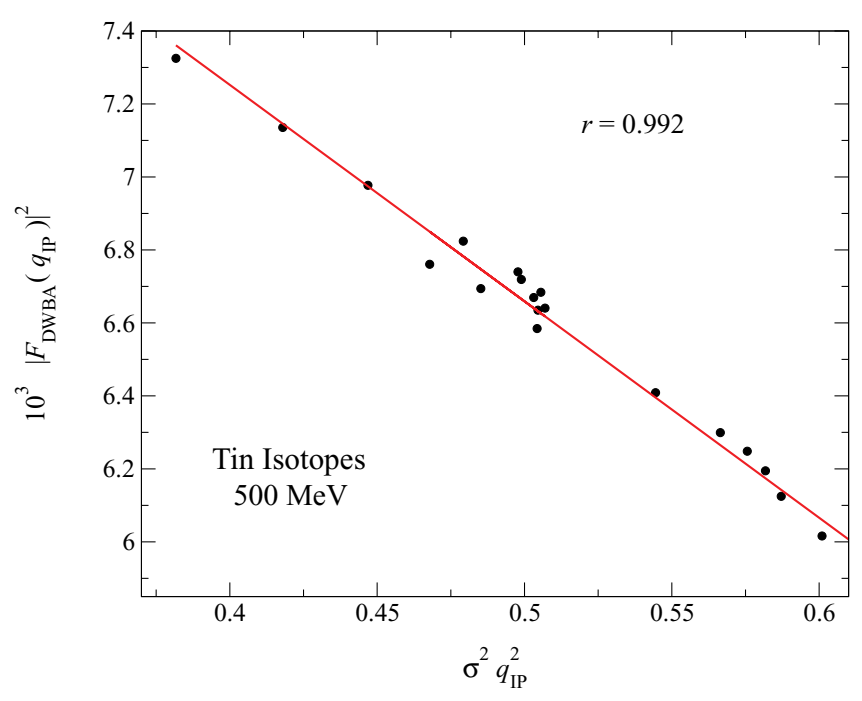

FIG. 9. (Color online) Correlation along the $\mathrm{Sn}$ isotopic chain of the squared charge form factor in DWBA, at its first inflection point $\left(q_{\mathrm{IP}}\right)$, with the product of the Helm parameter $\sigma^{2}$ and $q_{\mathrm{IP}}^{2}$. The results are computed with the $G 2$ effective interaction for scattering by $500-\mathrm{MeV}$ electrons. Note that each point of the figure corresponds to a different isotope and that the mass number increases from right to left.

Helm parameter $R_{0}$ in the tin isotopic chain:

$$
R_{0} \approx \frac{4.934}{q_{\mathrm{IP}}}
$$

We also find that the rms charge radii of the tin isotopes exhibit a considerable linear correlation with the momentum transfer $q_{\mathrm{IP}}$, as depicted in the lower panel of Fig. 8. These correlations could, in principle, provide an alternative way to obtain the parameter $\sigma$ of the equivalent Helm charge densities directly through Eq. (18), taking into account the relationship (23) between $R_{0}$ and $q_{\mathrm{IP}}$.

Figure 9 displays the evolution in the $\mathrm{Sn}$ chain of $\left|F_{\text {DWBA }}\left(q_{\mathrm{IP}}\right)\right|^{2}$ (the value of the DWBA squared charge form factor calculated at $\left.q_{\mathrm{IP}}\right)$ as a function of $\sigma^{2} q_{\mathrm{IP}}^{2}$. The addition of neutrons along the isotopic chain in general brings about an increase of the value of $\left|F_{\mathrm{DWBA}}\left(q_{\mathrm{IP}}\right)\right|^{2}$, also documented in the literature [20,21,23-25]. Furthermore, we notice that an interesting linear correlation arises between the quantities $\left|F_{\mathrm{DWBA}}\left(q_{\mathrm{IP}}\right)\right|^{2}$ and $\sigma^{2} q_{\mathrm{IP}}^{2}$ as one moves from the proton-rich side of the isotopic chain to the neutron-rich side. This correlation may be qualitatively understood in the following terms. If for guidance we consider the expression (19) of the electric charge form factor in the adopted Helm model, we see that the natural variables to investigate the variation of the charge form factor are $q R_{0}$ and $\sigma^{2} q^{2}$. But, as stated in Eq. (23), the value of $q R_{0}$ at the first inflection point of $\left|F_{\mathrm{DWBA}}(q)\right|^{2}$ is practically independent of the mass number in the whole isotopic chain. Therefore, the mass-number variation of $\sigma^{2} q_{\mathrm{IP}}^{2}$ is left as the principal source for the $A$ dependence of the value of the squared charge form factor at $q=q_{\mathrm{IP}}$. It is then reasonable that the change with $A$ of $\left|F_{\mathrm{DWBA}}\left(q_{\mathrm{IP}}\right)\right|^{2}$ and $\sigma^{2} q_{\mathrm{IP}}^{2}$ is correlated along the isotopic chain, a feature confirmed by Fig. 9. As a physical insight, helped by Eq. (23), the product
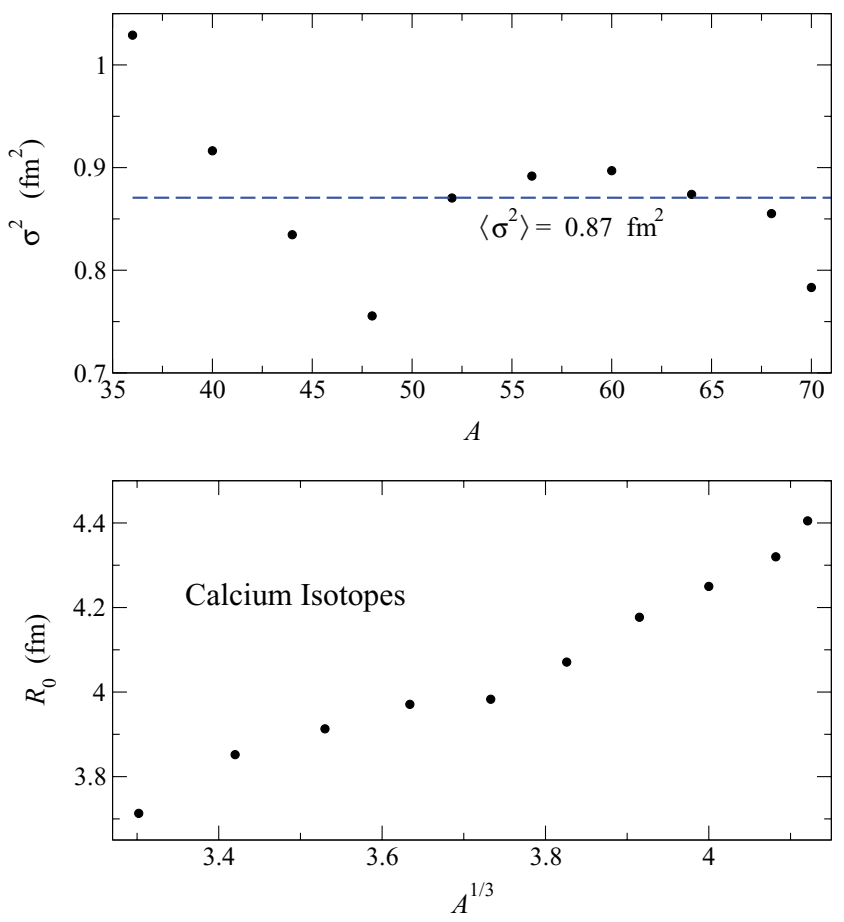

FIG. 10. (Color online) Same as described in the caption to Fig. 6 for the $\mathrm{Ca}$ isotopic chain.

$\sigma^{2} q_{\mathrm{IP}}^{2}$ can be recast as proportional to $\sigma^{2} / R_{0}^{2}$, which is the ratio between the surface width and the mean location of the surface of the underlying nuclear charge density.

Similar results to those discussed above for tin have been obtained in the study of the calcium isotopic chain. They are presented in Figs. 10-13. We first have modeled the
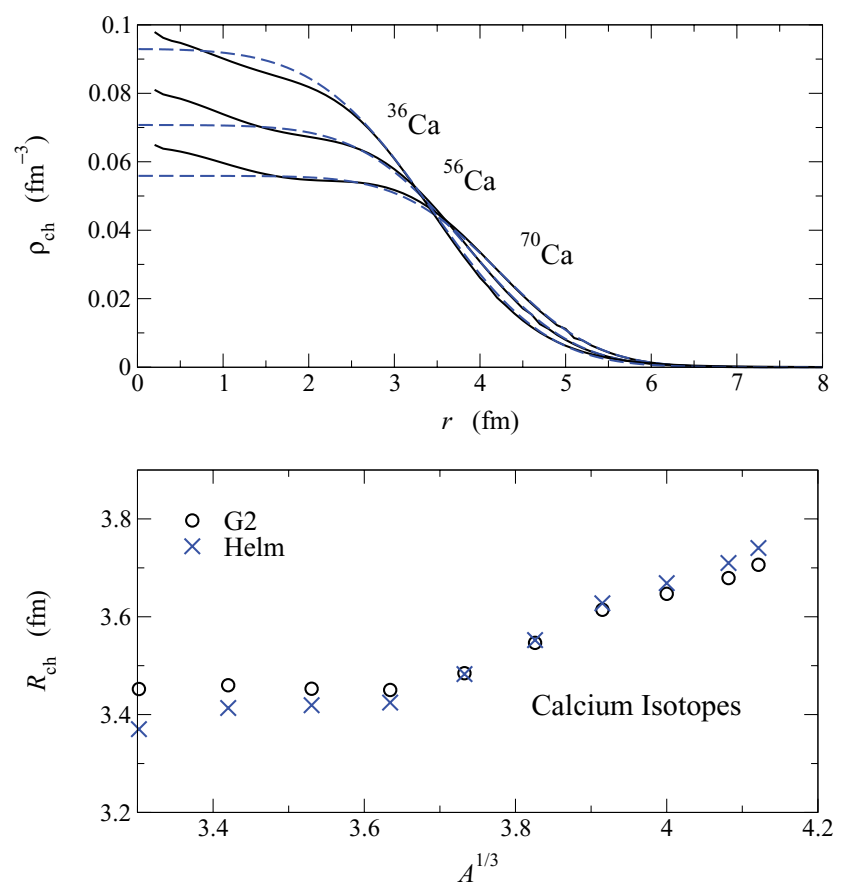

FIG. 11. (Color online) Same as described in the caption to Fig. 7 for the $\mathrm{Ca}$ isotopic chain. 

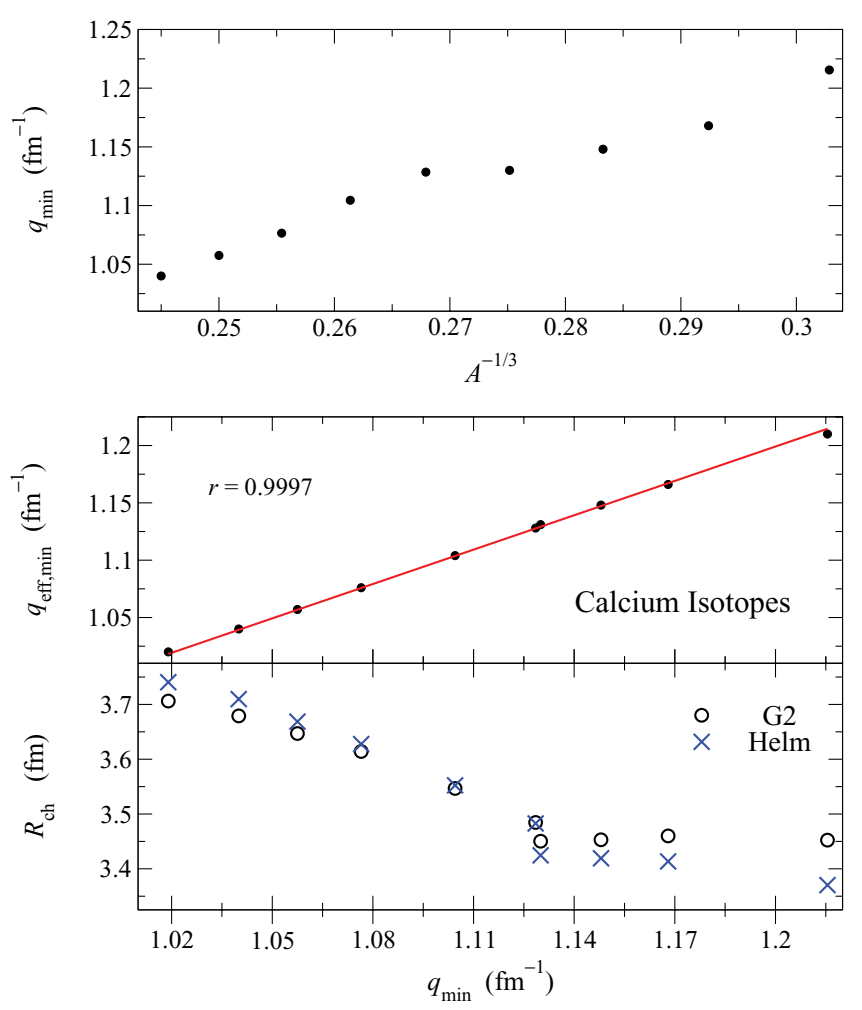

FIG. 12. (Color online) Same as described in the caption to Fig. 8 for the $\mathrm{Ca}$ isotopic chain.

calculated mean-field densities of the calcium chain by the simpler form of Helm densities. The mass-number evolution of the parameters of the Helm charge densities is illustrated in Fig. 10. The shrinkage of the Helm parameter $\sigma$ at magic neutron numbers, noticed in the tin chain, is a feature also present in the calcium isotopes for the magic neutron numbers $N=28$ and 50. However, the effect at $N=20$ is completely washed out, in agreement with the result of Ref. [67]. The mean-field charge densities of ${ }^{36} \mathrm{Ca},{ }^{56} \mathrm{Ca}$, and ${ }^{70} \mathrm{Ca}$ calculated

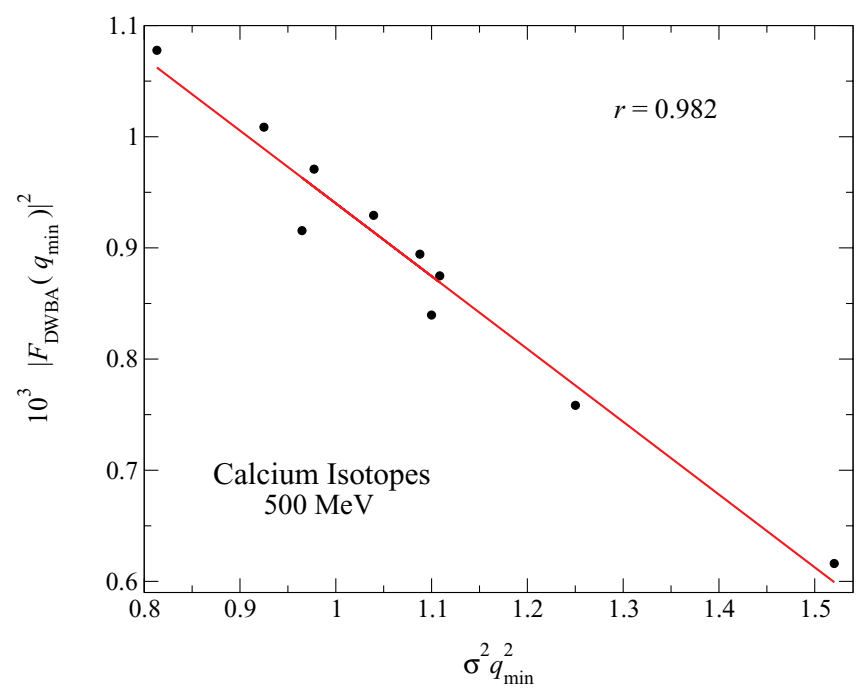

FIG. 13. (Color online) Same as described in the caption to Fig. 9 for the $\mathrm{Ca}$ isotopic chain. with $G 2$, along with the equivalent Helm profiles, are displayed in the upper panel of Fig. 11. As in the case of the tin chain, the effects of the addition of neutrons are very manifest in the mean-field charge density, as one can appreciate from the changes in the interior and surface regions of the density distributions of the shown $\mathrm{Ca}$ isotopes. One also sees that the Helm densities manage to follow on average these changes. The Helm profiles are found to reproduce closely the surface region of the mean-field densities, including the isotopes at the proton- and neutron-rich sides of the calcium chain. Discrepancies are observed in the interior region of the density distributions. In the lower panel of Fig. 11 we display, as a function of $A^{1 / 3}$, the variation in the calcium chain of the rms radii of the mean-field and Helm charge densities. Compared to the case of $\mathrm{Sn}$, this lighter chain presents more significant departures from the $A^{1 / 3}$ behavior. Also, the agreement of the rms radii of the Helm model with the self-consistent $G 2$ values is less good. This is more visible in approaching the drip lines, especially at the proton drip line.

Analogous correlations to those previously discussed in the case of tin, between the value of $q$ at the first minimum (or IP) of $\left|F_{\text {DWBA }}(q)\right|^{2}$ with (i) the mass number, (ii) the first minimum of $\left|F_{\mathrm{PWBA}}(q)\right|$, and (iii) the rms radius of the charge distributions, are similarly found in the analysis of the calcium isotopes. They are displayed in Fig. 12. In the present case, however, a significant departure of the radii $R_{0}$ and $R_{\mathrm{ch}}$ from the $A^{1 / 3}$ law is observed as one moves toward the proton drip line. These deviations may be largely due to the fact that, in approaching the proton drip line, the protons are more loosely bound and therefore the charge density extends to larger distances compared with the stable nuclei above ${ }^{40} \mathrm{Ca}$. The effect is much more prominent in calcium than in tin because of the lower Coulomb barrier due to its smaller atomic number. In turn, the same effect may originate that the Helm parameter $\sigma$ of ${ }^{40} \mathrm{Ca}$, measuring the surface thickness of the nucleus, does not decrease as compared with the heavier neighbor nuclei (see upper panel of Fig. 10). We plot in Fig. 13 the square of the DWBA charge form factor computed for 500-MeV electrons at its first minimum against the value of the product $\sigma^{2} q_{\min }^{2}$. In calcium, as in the case of the tin isotopes, an outstandingly linear correlation exists between both quantities.

Finally, to validate the consistency of our analysis with Helm density equivalents, we compute in test cases the squared DWBA electric charge form factor both with the Helm profiles and with their original mean-field charge densities. The results, as a function of the momentum transfer $q$, in a few isotopes of calcium and tin are compared in Fig. 14. The cases shown in the figure are chosen to illustrate the situation from the proton to the neutron drip lines, but similar conclusions are found for the other isotopes of these chains. In the range of $q$ values up to $\approx 1.5 \mathrm{fm}^{-1}$ in the calcium isotopes and up to around $\approx 1 \mathrm{fm}^{-1}$ in the tin isotopes, one observes an excellent agreement between the results for $\left|F_{\mathrm{DWBA}}(q)\right|^{2}$ obtained using the mean-field charge densities and using the equivalent Helm charge densities. A similar situation is found in the nuclei that lie near the drip lines: the agreement between the Helm and the mean field results is slightly worse but without sizable differences compared to the more stable nuclei. This scenario 

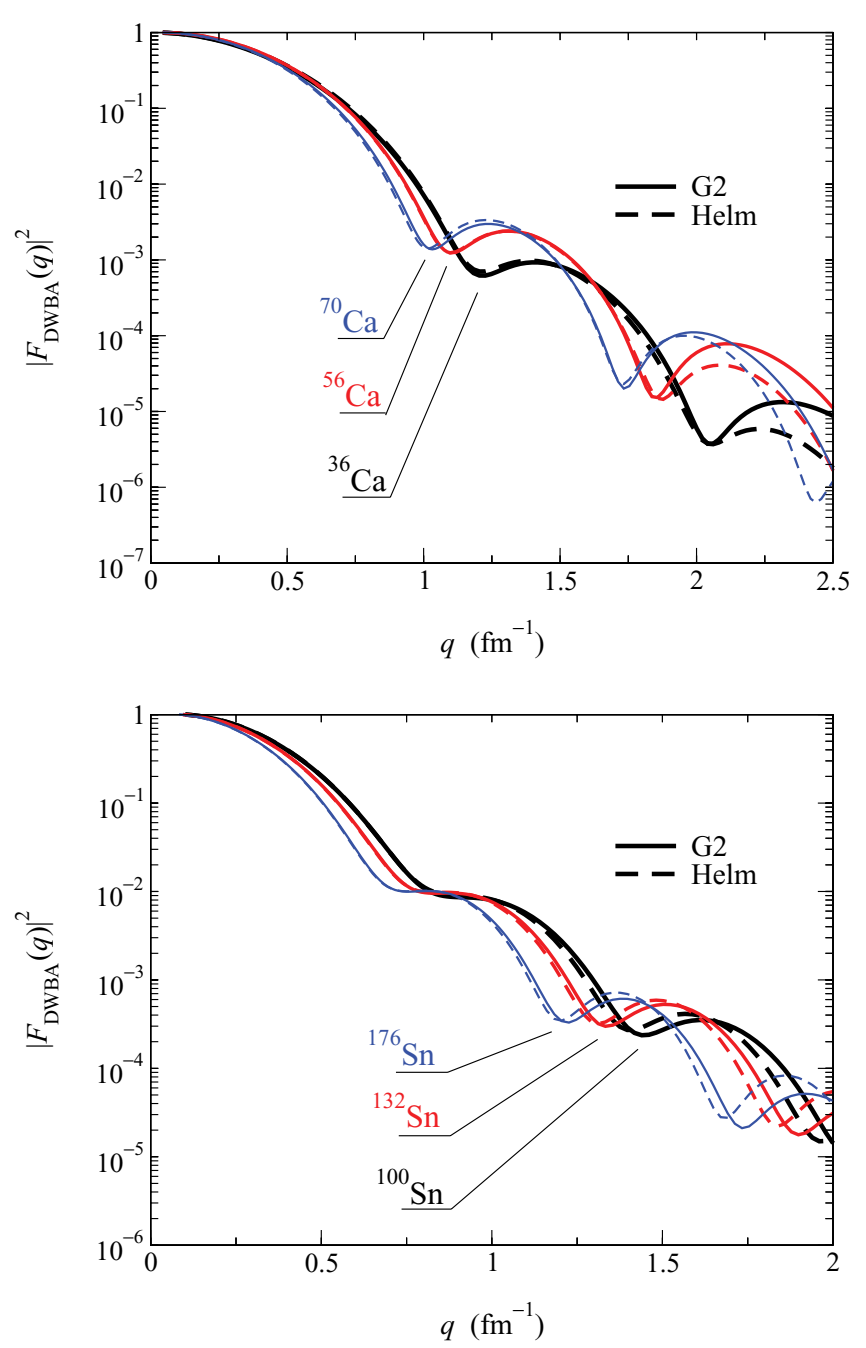

FIG. 14. (Color online) Comparison in $\mathrm{Ca}$ and $\mathrm{Sn}$ isotopes between the squared charge form factor in DWBA calculated with the charge densities of the $G 2$ mean-field interaction (solid lines) and with the equivalent Helm charge densities (dashed lines). Notice that the vertical and horizontal scales for calcium and tin are different.

points out that the electron-nucleus scattering results in the low-momentum transfer region computed with mean-field charge densities, are very well simulated by equivalent Helm densities with the $R_{0}$ and $\sigma$ parameters obtained according to the method described above. As the value of the momentum transfer increases, expectably, the discrepancies between the actual calculations with $G 2$ and the adjusted Helm model become evident.

Let us now concentrate on the results pertaining to the self-consistent calculations with $G 2$ that are shown in Fig. 14. One can observe that, as mentioned earlier, the first minimum that is visible in the squared charge form factor of calcium sinks away in tin. Its fingerprint in tin is recognized as an inflection point. The second and further minima of $\left|F_{\mathrm{DWBA}}(q)\right|^{2}$ remain, however, clearly identifiable in the tin isotopes. With the progressive addition of neutrons in either of the two isotopic chains, it may be seen from Fig. 14 that the first minimum of $\left|F_{\text {DWBA }}(q)\right|^{2}$, or its signature, becomes slightly more marked.
This effect is, however, much less noticeable than the discussed effect on the first minimum induced by changing $Z$. One also observes that the location of the minima or inflection points of the squared charge form factor is gradually pushed toward lower-momentum transfers as the nucleus becomes more and more neutron rich. This effect has been noted in previous literature [20,21,23-25], and for the first minimum we have described it in more detail in the upper panels of Figs. 8 and 12. The same trend (inwards shift of the minima or IP) occurs with increasing atomic number (compare the scales of the $q$ axis for $\mathrm{Ca}$ and $\mathrm{Sn}$ in Fig. 14). In general, the inwards displacement of the momentum transfer of the minima is accompanied by a simultaneous increase of the height of the maxima of the squared charge form factor.

\section{SUMMARY AND CONCLUSIONS}

We adapted the ELSEPA code [30] for calculations of elastic electron-nucleus scattering. The predictions obtained from the charge densities computed with various selected Skyrme forces and modern relativistic mean-field parameter sets have been compared with existing experimental data about elastic electron scattering in several stable nuclei.

A suitable quantitative comparison among the theoretical predictions is established by introducing, for each nucleus and beam energy, a normalized square weighted difference. Although all the considered effective interactions describe qualitatively well the experimental DCS data, the quantitative analysis in terms of the $d_{w}^{2}$ with respect to experiment shows some differences among the various theoretical DCS, especially for large scattering angles. These differences are related mainly with the different behavior of the mean-field densities in the inner region of the nuclei. For the investigated nuclei and energies, one always finds a theoretical charge distribution whose $d_{w}^{2}$ is at least similar, and in some cases better, than the one obtained with the experimental charge density that has been fitted to reproduce the measured DCS data. Among the nuclear interactions analyzed, the DCS values calculated with the charge densities obtained from the covariant DD-ME2 and FSUGold parameter sets are the ones that tend to give overall better agreement with the experimental values considered.

A more challenging test on theory is provided by the analysis of the experimental data about relative differences of DCS between pairs of neighbor nuclei. The calculations based on the mean-field charge densities reasonably follow the global trends shown up by the experimental measurements. The quantitative analysis, however, as in the case of the previously investigated DCS, highlights some deficiencies of the mean-field densities in their inner region.

We have used the mean-field charge densities obtained with the relativistic $G 2$ parametrization, by the reasons discussed previously, as the input baseline to study the elastic electronnucleus scattering along the tin and calcium isotopic chains. Calculations have been performed from the proton to the neutron drip lines. Our aim has been to extract general trends, according to current mean-field theories of nuclear structure, about the behavior that may be expected from real 
electron-nucleus scattering experiments in exotic nuclei, in the low-momentum transfer region. Such experiments are envisaged at FAIR [17] and the SCRIT [18,19] project in the nearby future, using exotic nuclei provided by radioactive isotope beams. We have confined our study to medium and heavy mass isotopes where the mean-field approach can be applied. Surely, the experimental program will investigate not only heavy exotic nuclei but it will address also scattering from light exotic nuclei. The theoretical investigation of these light nuclei, however, demands a sophisticated microscopic treatment of scattering to deal with the underlying shell-model structure and the possible occurrence of halos, which is beyond the methodology of the mean-field study of the present work.

First, we have computed for each isotope of the investigated chains the squared electric charge form factor. It has been obtained as the ratio between the DWBA DCS calculated with ELSEPA and the Mott DCS. We have checked that $\left|F_{\text {DWBA }}(q)\right|^{2}$ defined in this way is relatively independent of the energy of the electron beam up to momentum transfers $q \approx 1-$ $1.5 \mathrm{fm}^{-1}$, even for nuclei as large as ${ }^{208} \mathrm{~Pb}$. Second, we have fitted the mean-field charge densities by two-parameter Helm distributions. In doing so, we have adjusted the effective momentum transfer correction to reproduce the mean-field rms charge radii, on average, along the isotopic chain. We have made an a posteriori check that a DWBA calculation of the elastic electron scattering using as input the equivalent Helm charge density, is in excellent agreement with the results computed with the original mean-field charge density up to momentum transfers $q \approx 1-1.5 \mathrm{fm}^{-1}$.

We have paid special attention to the value of the square of the DWBA electric charge form factor at the momentum transfer where its first minimum, in medium-mass nuclei, or its first inflection point, in heavier nuclei, appears. We have studied how it evolves along the isotopic chains of tin and calcium. Interesting linear correlations between the value of the momentum transfer at the first minimum (or IP) of $\left|F_{\mathrm{DWBA}}(q)\right|^{2}$ with the mass number of the isotopes of the chain, with the effective momentum transfer at the first minimum of $\left|F_{\mathrm{PWBA}}(q)\right|^{2}$, and with the rms radius of the charge distribution, have been discussed. Also, a linear correlation between $\left|F_{\text {DWBA }}(q)\right|^{2}$ and $\sigma^{2} q^{2}$ computed at the first minimum or IP, where $\sigma$ is the Helm parameter that accounts for the surface thickness of the nuclear density, has been found in the studied chains.

The analysis described in the present article could potentially be useful for future electron-nucleus elastic-scattering experiments. If the experimental data are available for two or more isotopes of a given chain, the aforementioned linear correlations would provide, for an unknown nucleus of the chain, a hint on the value expected for the square of the experimental electric charge form factor at its first minimum and for the momentum transfer where the latter occurs. The parameters of the Helm charge density distribution of the unknown isotope could be estimated by means of correlations such as those displayed in Figs. 8 and 12, with the help of Eqs. (20) and (18). Also, if the value of the squared modulus of the form factor is determined experimentally at its first minimum, the charge density in the Helm model can be sketched from similar correlations to Figs. 8 and 12, together with the correlation of the type depicted in Figs. 9 and 13. The use of more elaborated versions of the Helm model [67-69] that take into account the central depression of the charge density should allow one to extend the domain of validity of our method up to larger values of the momentum transfer. Work in this direction will be undertaken.

\section{ACKNOWLEDGMENTS}

We are very grateful to Professor P. Ring for supplying us with the code for finite nuclei calculations with the model DD-ME2. We thank Professor D. W. L. Sprung for reading a preliminary version of the manuscript and for valuable comments. This work has been partially supported by Grants Nos. FIS2005-03142 and FPA2006-12066 from MEC (Spain) and FEDER and by Grant No. 2005SGR-00343 from Generalitat de Catalunya, as well as by the Spanish ConsoliderIngenio 2010 Programme CPAN CSD2007-00042. One of us (X.R.) also acknowledges Grant No. AP2005-4751 from MEC (Spain).
[1] R. Hofstadter, Rev. Mod. Phys. 28, 214 (1956).

[2] T. W. Donnelly and J. D. Walecka, Annu. Rev. Nucl. Part. Sci. 25, 329 (1975).

[3] T. W. Donnelly and I. Sick, Rev. Mod. Phys. 56, 461 (1984).

[4] E. Moya de Guerra, Phys. Rep. 138, 293 (1986).

[5] I. Sick, Prog. Part. Nucl. Phys. 47, 245 (2001).

[6] H. de Vries, C. W. de Jager, and C. de Vries, At. Data Nucl. Data Tables 36, 495 (1987).

[7] G. Fricke, C. Bernhardt, K. Heiling, L. A. Schaller, L. Shellenberg, E. B. Shera, and C. W. de Jager, At. Data Nucl. Data Tables 60, 177 (1995).

[8] I. Angeli, At. Data Nucl. Data Tables 87, 185 (2004).

[9] Proceedings of the ENAM'04 Conference, Pine Mountain (USA), 2004, edited by C. J. Gross, W. Nazarewicz, and K. P. Rykaczewski, Eur. Phys. J. A 25, s01 (2005).

[10] I. Tanhihata, Prog. Part. Nucl. Phys. 35, 505 (1995).
[11] H. Geissel, G. Müzenberg, and R. Riisager, Annu. Rev. Nucl. Part. Sci. 45, 163 (1995).

[12] A. Mueller, Prog. Part. Nucl. Phys. 46, 359 (2001).

[13] T. Suda, K. Maruyama, and I. Tanihata, RIKEN Accel. Prog. Rep. 34, 49 (2001).

[14] K. Katayama, T. Suda, and I. Tanihata, Phys. Scr., T 104, 129 (2003).

[15] An International Accelerator Facility for Beams of Ions and Antiprotons, GSI report 2006. http://www.gsi.de/GSI-Future/cdr/.

[16] H. Simon, in Proceedings of the International Workshop XXXII on Gross Properties of Nuclei and Nuclear Excitations, Hirschegg (Austria), 2004, edited by M. Buballa, J. Knoll, W. Nörenberg, B.-J. Schaefer, and J. Wambach (GSI, Darmstad, 2004), p. 290.

[17] H. Simon, Nucl. Phys. A787, 102 (2007).

[18] T. Suda and M. Wakasugi, Prog. Part. Nucl. Phys. 55, 417 (2005). 
[19] M. Wakasugi, T. Emoto, Y. Furukawa, K. Ishii, S. Ito, T. Koseki, K. Kurita, A. Kuwajima, T. Masuda, A. Morikawa, M. Nakamura, A. Noda, T. Ohnishi, T. Shirai, T. Suda, H. Takeda, T. Tamae, H. Tongu, S. Wang, and Y. Yano, Phys. Rev. Lett. 100, 164801 (2008).

[20] A. N. Antonov, D. N. Kadrev, M. K. Gaidarov, E. Moya de Guerra, P. Sarriguren, J. M. Udias, V. K. Lukyanov, E. V. Zemlyanaya, and G. Z. Krumova, Phys. Rev. C 72, 044307 (2005).

[21] P. Sarriguren, M. K. Gaidarov, E. M. de Guerra, and A. N. Antonov, Phys. Rev. C 76, 044322 (2007).

[22] S. Karataglidis and K. Amos, Phys. Lett. B650, 148 (2007).

[23] C. A. Bertulani, J. Phys. G 34, 315 (2007).

[24] Zaijun Wang and Zhongzhou Ren, Phys. Rev. C 70, 034303 (2004).

[25] Zaijun Wang and Zhongzhou Ren, Phys. Rev. C 71, 054323 (2005).

[26] Zaijun Wang, Zhongzhou Ren, and Ying Fan, Phys. Rev. C 73, 014610 (2006).

[27] K. Amos, S. Karataglidis, and J. Dobaczewski, Phys. Rev. C 70, 024607 (2004).

[28] A. Baker, Phys. Rev. 134, B240 (1964).

[29] D. R. Yennie, D. G. Ravenhall, and R. N. Wilson, Phys. Rev. 95, 500 (1954).

[30] F. Salvat, A. Jabalonski, and C. J. Powell, Comput. Phys. Commun. 165, 157 (2005).

[31] J. Bartel, P. Quentin, M. Brack, C. Guet, and H.-B. Håkansson, Nucl. Phys. A386, 79 (1982); M. Brack, C. Guet, and H.-B. Håkansson, Phys. Rep. 123, 275 (1985).

[32] E. Chabanat, P. Bonche, P. Haensel, J. Meyer, and R. Schaeffer, Nucl. Phys. A635, 231 (1998).

[33] G. A. Lalazissis, J. König, and P. Ring, Phys. Rev. C 55, 540 (1997).

[34] B. G. Todd-Rutel and J. Piekarewicz, Phys. Rev. Lett. 95, 122501 (2005).

[35] R. J. Furnstahl, B. D. Serot, and H.-B. Tang, Nucl. Phys. A615, 441 (1997); [Erratum-ibid. A640, 505 (1998)].

[36] T. Niksić, D. Vretenar, P. Finelli, and P. Ring, Phys. Rev. C 66, 024306 (2002).

[37] W. Greiner and J. Reinhardt, Quantum Electrodynamics (Springer-Verlag, Berlin/Heidelberg, 1992).

[38] B. D. Serot and J. D. Walecka, Int. J. Mod. Phys. E 6, 515 (1997).

[39] W. Bertozzi, J. Friar, J. Heisenberg, and J. W. Negele, Phys. Lett. B41, 408 (1972).

[40] E. Garrido and E. Moya de Guerra, Nucl. Phys. A650, 387 (1999).
[41] UNEDF SciDAC Collaboration, http://www.unedf.org.

[42] M. Bender, P.-H. Heenen, and P.-G. Reinhard, Rev. Mod. Phys. 75, 121 (2003).

[43] D. Vautherin and D. M. Brink, Phys. Rev. C 5, 626 (1972).

[44] Li Guo-Quiang, J. Phys. G 17, 1 (1991).

[45] W. A. Richter and B. A. Brown, Phys. Rev. C 67, 034317 (2003).

[46] B. D. Serot and J. D. Walecka, Adv. Nucl. Phys. 16, 1 (1986).

[47] P.-G. Reinhard, Rep. Prog. Phys. 52, 439 (1989).

[48] B. D. Serot, Rep. Prog. Phys. 5, 1855 (1992).

[49] Y. K. Gambhir, P. Ring, and A. Thimet, Ann. Phys. (NY) 198, 132 (1990).

[50] P. Ring, Prog. Part. Nucl. Phys. 37, 193 (1996).

[51] J. Boguta and A. R. Bodmer, Nucl. Phys. A292, 413 (1977).

[52] Y. Sugahara and H. Toki, Nucl. Phys. A579, 557 (1994).

[53] M. Del Estal, M. Centelles, X. Viñas, and S. K. Patra, Phys. Rev. C 63, 024314 (2001).

[54] C. J. Horowitz and J. Piekarewicz, Phys. Rev. Lett. 86, 5647 (2001); Phys. Rev. C 64, 062802(R) (2001).

[55] P. Arumugam, B. K. Sharma, P. K. Sahu, S. K. Patra, Tapas Sil, M. Centelles, and X. Viñas, Phys. Lett. B601, 51 (2004).

[56] M. Del Estal, M. Centelles, X. Viñas, and S. K. Patra, Phys. Rev. C 63, 044321 (2001).

[57] M. E. Rose, Relativistic Electron Theory (Wiley, New York, 1961).

[58] F. Salvat, J. M. Fernandez-Varea, and W. Williamson Jr., Comput. Phys. Commun. 90, 151 (1995).

[59] F. Salvat and R. Mayol, Comput. Phys. Commun. 74, 358 (1993).

[60] I. Sick and J. S. McCarthy, Nucl. Phys. A150, 631 (1970).

[61] R. F. Frosch, R. Hofstadter, J. S. McCarthy, G. K. Nöldeke, K. J. van Oostrum, M. R. Yearian, B. C. Clark, R. Herman, and D. G. Ravenhall, Phys. Rev. 174, 1380 (1968).

[62] Phan Xuan Ho, J.-B. Bellicard, A. Bussiere, Ph. Leconte, and M. Priou, Nucl. Phys. A179, 529 (1972).

[63] A. S. Litvinenko, N. G. Shevchenko, A. Yu. Buki, G. A. Savitsky, V. M. Khvastunov, A. A. Khomich, V. N. Polishchuk, and I. I. Chkalov, Nucl. Phys. A182, 265 (1972).

[64] J. L. Friar and J. W. Negele, Nucl. Phys. A212, 93 (1973).

[65] B. Dreher, J. Friedrich, K. Merle, H. Rothhaas, and G. Luhrs, Nucl. Phys. A235, 219 (1974).

[66] R. H. Helm, Phys. Rev. 104, 1466 (1956).

[67] J. Friedrich and N. Voegler, Nucl. Phys. A373, 192 (1982).

[68] J. Friedrich, N. Voegler, and P. G. Reinhard, Nucl. Phys. A459, 10 (1986).

[69] D. C. Zheng, N. Yamanishi, and D. W. L. Sprung, Nucl. Phys. A550, 89 (1992). 